University of Louisville

ThinkIR: The University of Louisville's Institutional Repository

Electronic Theses and Dissertations

$12-2019$

\title{
Characterizing the roles of neutral ceramidase in cisplatin- induced kidney injury.
}

Sophia M. Sears

University of Louisville

Follow this and additional works at: https://ir.library.louisville.edu/etd

Part of the Pharmacology, Toxicology and Environmental Health Commons

\section{Recommended Citation}

Sears, Sophia M., "Characterizing the roles of neutral ceramidase in cisplatin-induced kidney injury." (2019). Electronic Theses and Dissertations. Paper 3396.

https://doi.org/10.18297/etd/3396

This Master's Thesis is brought to you for free and open access by ThinkIR: The University of Louisville's Institutional Repository. It has been accepted for inclusion in Electronic Theses and Dissertations by an authorized administrator of ThinkIR: The University of Louisville's Institutional Repository. This title appears here courtesy of the author, who has retained all other copyrights. For more information, please contact thinkir@louisville.edu. 


\title{
CHARACTERIZING THE ROLES OF NEUTRAL CERAMIDASE IN CISPLATIN-
} INDUCED KIDNEY INJURY

\author{
By \\ Sophia M. Sears \\ B.A. Molecular Biology \& Biochemistry, Goshen College, 2017
}

A Thesis submitted to the faculty of the School of Medicine of the University of Louisville in partial fulfillment of the requirements for the degree of

Masters of Science in Pharmacology and Toxicology

Department of Pharmacology and Toxicology

University of Louisville

Louisville, Kentucky

December 2019 

CHARACTERIZING THE ROLES OF NEUTRAL CERAMIDASE IN CISPLATININDUCED KIDNEY INJURY

\author{
By \\ Sophia M. Sears \\ B.A. Molecular Biology \& Biochemistry, Goshen College, 2017
}

A Thesis approved on

August 15, 2019

By the following thesis committee:

Leah Siskind, Ph.D.

Levi Beverly, Ph.D.

Geoff Clark, Ph.D.

Eleanor Lederer, M.D.

Kavitha Yaddanapudi, Ph.D. 


\section{DEDICATION}

This thesis is dedicated to my friends and family who supported me as a developing scientist and always pushed me to be my best. 


\section{ACKNOWLEGMENTS}

I would like to thank my lab mates for helping me physically and emotionally through this project. I would also like to thank Drs. Siskind and Beverly for guiding me in my research and providing valuable feedback. 


\begin{abstract}
CHARACTERIZING THE ROLES OF NEUTRAL CERAMIDASE IN CISPLATININDUCED KIDNEY INJURY
\end{abstract}

Sophia M. Sears

August 15, 2019

Cisplatin is a commonly used chemotherapeutic agent with a dose-limiting nephrotoxicity. $30 \%$ of patients given cisplatin develop acute kidney injury (AKI). AKI increases risk of chronic kidney disease (CKD) development and mortality. Patients that don't develop clinical AKI are still at risk for long term declines in renal function. Currently, there are no FDA approved agents to treat or prevent cisplatin-induced kidney injury (CDDP-KI). In this study, we demonstrated that neutral ceramidase (nCDase) knockout provides protection from AKI in the highdose model of CDDP-KI. However, in the repeated low dose cisplatin (RLDC) model of injury and we found nCDase knockout does not prevent development of CKD. We also observed that $\mathrm{nCDase}$ knockout reduces induction of ER stress in the single high-dose model but not in the RLDC model. This study suggests there are unique mechanisms of RLDC induced kidney injury which may have hindered development of nephroprotective agents. 


\section{TABLE OF CONTENTS}

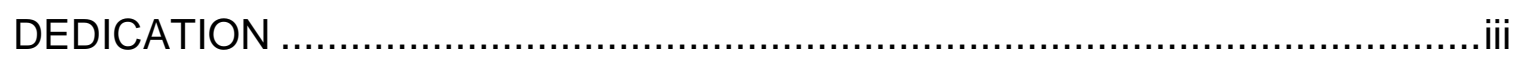

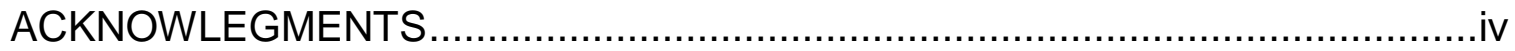

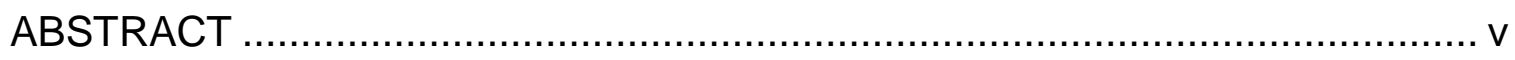

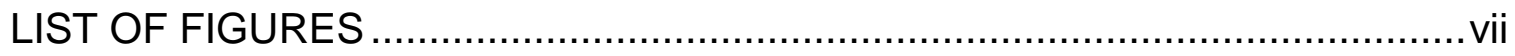

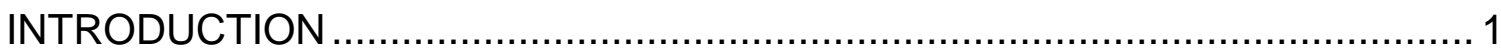

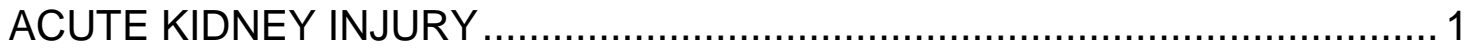

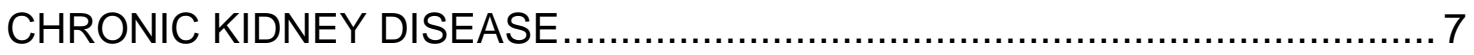

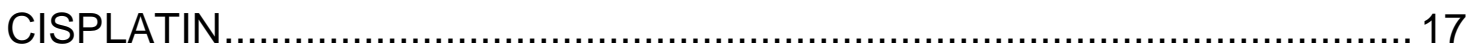

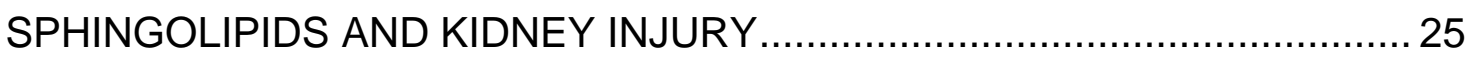

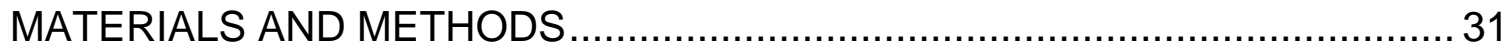

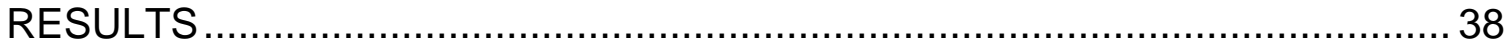

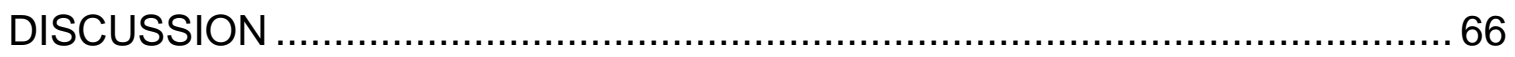

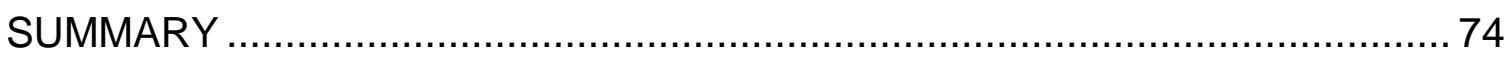

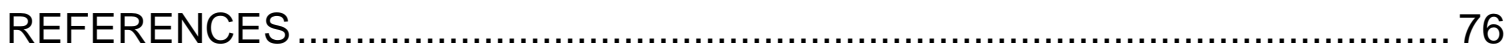

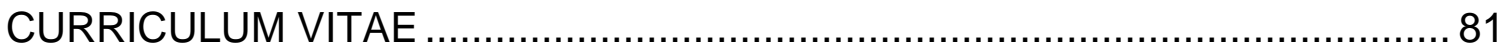




\section{LIST OF FIGURES}

Figure 1. Viability of nCDase knockout and wild type MEFs during FBS withdrawal...... 39

Figure 2. Upregulation of $p-A K T$ and inhibition of GSK3 $\beta$ are induced by nCDase knockout. 41

Figure 3. Viability and nCDase enzymatic activity of wild type MEFs treated with pharmacologic inhibitors of nCDase. 44

Figure 4. Acute inhibition of nCDase confers signaling changes as seen in nCDase knockout MEFs. 45

Figure 5. nCDase -/- mice display increased LC3 expression compared to wild type.

Figure 6. Loss of nCDase attenuates AKI following high-dose cisplatin treatment.

Figure 7. Loss of $\mathrm{nCDase}$ protects from high-dose cisplatin-induced deterioration in kidney pathology. 49

Figure 8. Loss of nCDase decreases mRNA expression of inflammatory cytokines in the kidney following high-dose cisplatin treatment. 50

Figure 9. Loss of nCDase attenuates high-dose cisplatin-induced apoptosis..... 52 Figure 10. Loss of nCDase attenuates high-dose cisplatin-induced ER stress... 54 Figure 11. RLDC treatment of C57BL/6 mice induces changes in protein expression similar to those triggered by $\mathrm{nCDase}$ inhibition in vitro. 56 
Figure 12. nCDase -/- does not prevent RLDC induced kidney injury.

Figure 13. Loss of nCDase does not protect from RLDC induced renal pathology. 60

Figure 14. nCDase inhibition does not alter inflammatory cytokine production or

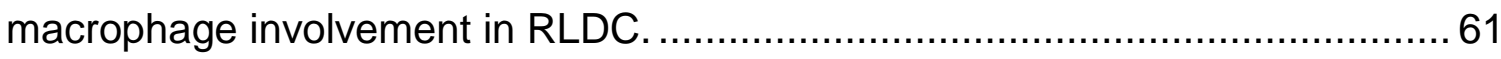

Figure 15. nCDase inhibition does not alter fibronectin expression or collagen deposition following RLDC. 63

Figure 16. nCDase -/- mice are not protected from ER stress in RLDC. 65 


\section{INTRODUCTION}

\section{ACUTE KIDNEY INJURY \\ Epidemiology and Defining AKI}

Acute kidney injury (AKI) is defined as a rapid decrease in renal function characterized by a decrease in glomerular filtration rate (GFR) and an increase in retained waste products.[1] It is one of the most common life-threatening conditions associated with hospital admissions. Since 2002, it has been estimated that $25 \%$ of patients admitted to the hospital develop AKI. [2, 3] Development of AKI increases risk of mortality 5.5-6.5 fold compared to similarly ill patients who do not develop AKI.[4]

As our population ages, incidence of $A K I$ is increasing. Age alone increases the likelihood of AKI development, but the increase in complications such as hypertension, cardiovascular disease, and chronic kidney disease also increase vulnerability to $\mathrm{AKI}$. Therapeutic interventions such as surgery and chemotherapy put patients at risk for AKI as well. All of these factors have contributed to the steadily increasing incidence of AKI, particularly among elderly hospitalized patients.[2] Financially, the rise in AKI cases is problematic. In 2005, the financial burden of $\mathrm{AKI}$ was estimated to be $\$ 10$ billion in additional cost to the health care system in the United States.[4]

The exact prevalence of AKI has been difficult to monitor due to its broad definition and variable methods of measuring renal function. An important 
function of the kidney is to filter out nitrogenous waste products from the blood to be excreted in the urine. A decline in GFR can therefore be observed by an increase in circulating nitrogenous compounds. Serum creatinine and blood urea nitrogen (BUN) are two products that can be measured to estimate GFR. Increased concentrations of these products in the blood corresponds to a decreased renal clearance. Therefore, serial measurements of serum creatinine and BUN can provide an idea of declining kidney function.[4] Until recently, there had not been set quantitative standards of kidney function that described an AKI diagnosis.

In order to provide more clarity, the RIFLE classification (Risk/Injury/Failure/Loss/End-stage renal disease) and AKIN classification (Acute Kidney Injury Network) were published providing measurable criteria to define stages of kidney injury based on measurements of serum creatinine and urine output.[2] The RIFLE scale classifies kidney injury cases by severity into categories of risk, injury, or failure. It further classifies outcomes of kidney injury as either loss of function or end stage renal disease. By RIFLE definitions, AKI corresponds to a doubling in patient serum creatinine from baseline and a 50\% reduction in GFR with urine output dropping below $0.5 \mathrm{ml} / \mathrm{kg} / \mathrm{hr}$ for 12 hours. Renal function must decline to these levels in 1 to 7 days and remain below these standards for at least 12 hours. $[5,6]$

In 2007, the AKIN classification was published to expand on the RIFLE classification. AKIN standards do not require estimation of baseline serum creatinine and considers diagnosis of AKI only after achieving adequate 
hydration and excluding urinary obstruction. AKI is diagnosed as stage 1,2 , or 3 based on the increase of serum creatinine within 48 hours, rather than from an estimated baseline. Stage 1, 2, and 3 on the AKIN scale corresponds to the risk, injury, and failure levels of RIFLE. Stage $2 \mathrm{AKI}$ is defined as an increase of 0.3 $\mathrm{mg} / \mathrm{dl}$ serum creatinine and a drop in urine output below $0.5 \mathrm{mg} / \mathrm{kg} / \mathrm{hr}$ for 12 hours.[5] In the clinic, patients are often diagnosed with AKI using a combination of RIFLE and AKIN classifications determined by the Kidney Disease Improving Global Outcomes (KDIGO) group. Diagnosis is based on either a $0.3 \mathrm{mg} / \mathrm{dl}$ increase of serum creatinine within 48 hours or a doubling of serum creatinine from estimated baseline occurring within 7 days.[5]

\section{Biomarkers of $\mathrm{AKI}$}

Although serum creatinine has been relied on in clinics for diagnosing AKI, it is not a perfect indicator. Serum creatinine does not become elevated until 2472 hours after a renal insult. It is also affected by factors such as age, race, sex, body weight, metabolism, and protein intake.[2] BUN also has limitations in determining GFR, as it is also affected by factors other than renal injury and spikes after renal damage has already occurred.[4] As the incidence of AKI development is increasing with an aging population, it has become more and more important to develop new biomarkers with more sensitivity and specificity. Ideally, new biomarkers would be more specific to kidney damage and would indicate injury is occurring at earlier timepoints. Two newer biomarkers of AKI include neutrophil gelatinase-associated lipocalin (NGAL) and kidney injury marker 1 (Kim-1). 
NGAL gained attention as a biomarker of AKI when it was observed to be elevated in the kidney following ischemic and nephrotoxic insults in animal studies.[7] It was first identified in neutrophils, but it also functions as a rapid response protein to tissue injury. NGAL has antibacterial effects through iron scavenging properties and promotes epithelial growth through an unknown mechanism.[8] NGAL has had mixed success in predicting AKI events clinically. Although it is a sensitive marker for kidney tissue damage, it has also been shown to elevate in other acute and chronic inflammatory conditions.

Development of an assay specific for kidney secreted NGAL would increase its specificity as a marker of AKI.[7, 8]

Kim-1 is a cell surface receptor in epithelial and lymphoid/myeloid cells. Its expression also increases significantly during AKI in proximal tubule cells.[8] It has been suggested that during AKI, proximal tubule cells lose their polarity and Kim-1 is secreted into the interstitium.[9] Although larger studies for validation are needed, Kim-1 shows promise as a specific and sensitive marker for subclinical AKI detection. It has been approved by the US FDA as a biomarker for preclinical drug development.[10]

\section{Pathology of AKI}

There are many underlying causes of AKI. AKI can arise due to prerenal, intrinsic, or postrenal conditions. Prerenal injury is due to decreased renal prefusion or volume depletion. Intrinsic injury is caused by direct damage or disruption within the kidneys. Postrenal injury occurs when there is insufficient urine drainage distal to the kidneys. While prerenal and postrenal injuries can 
often be corrected with repletion of intravascular volume or removal of a urinary blockage, intrinsic AKI must resolve with time and often requires renal replacement therapy such as dialysis.[11]

Intrinsic $\mathrm{AKI}$ is the most common form of $\mathrm{AKI}$ acquired in hospitalized patients, accounting for up to $70 \%$ of cases.[4] Intrinsic injury is divided into further categories based on where kidney damage is occurring. There are glomerular, interstitial, tubular, and vascular causes of intrinsic AKI. AKI is most often caused by acute tubular necrosis.[11] Eighty to ninety percent of the cases of acute tubular necrosis are a result of an ischemic or nephrotoxic insult. Despite the name, cell death is not always present in AKI caused by acute tubular necrosis and can include damage caused by sublethal changes in tubule cells. AKI involves several pathophysiological processes including endothelial damage and vascular impairment, immune response, and tubular cell death.[12] There processes orchestrate AKI development in phases of initiation, extension, maintenance, and recovery.[4]

In the initiation phase, the ischemic or nephrotoxic insult causes functional damage to tubule epithelial cells. Renal blood flow decreases as a result of injury, causing a decrease in available cellular ATP. This limited energy supply further damages epithelial and endothelial cells, triggering the release of cytokines and chemokines to initiate the inflammatory cascade. Epithelial cells will also undergo morphological changes, leading to a loss of the brush border membrane and begin undergoing apoptosis or necrosis. After death, these cells often slough off and cause tubular obstruction.[4] 
The extension phase defines the subsequent continued hypoxia and inflammatory response. Hypoxia is exacerbated by damage to renal vascular endothelial cells. Inflammatory and profibrotic cells begin infiltrating and proliferating in response to the earlier released signals. Tubule cells continue to die in this phase, leading to a continued decline in GFR.[4]

In the maintenance phase, repair processes begin. GFR halts its decline as tubule cells begin dedifferentiation, migration, and proliferation to re-establish structural integrity. Renal blood flow begins to return to normal. Cellular repair and reorganization begin the process of restoring organ function.[4]

In the recovery phase, renal function recovers. Epithelial cells re-establish polarity and return to normal function. Inflammatory and profibrotic cells are cleared. The extent to which renal function returns can vary depending on several factors. It is also hypothesized that there may be chronic impairment of vasculature and sublethal changes in tubule epithelial cells making the kidney more sensitive to additional insults.[4]

Episodes of AKI can lead to many different outcomes. Acute symptoms include disturbances in acid-base and electrolyte homeostasis, uremia, and fluid overload.[3] Currently, there are no FDA approved agents to prevent or treat AKI. Patients are often required to stay in the hospital to receive primarily supportive care. Patients' intravascular volume, electrolyte balance, and cardiac function are closely monitored. In certain cases, fluid replacement therapy can be used to correct imbalances.[11] 
Following an incident of $\mathrm{AKI}$, the kidney has the ability to repair tissue damage and return to normal function. However, long term studies have shown that patients who have suffered an AKI event have increased risk of developing chronic kidney disease (CKD), proteinuria, and cardiovascular mortality.[13] One study found that development of $\mathrm{AKI}$ requiring dialysis made patients 28 times more likely to develop CKD and doubled the risk of mortality.[14] As more research is done, the link between $\mathrm{AKI}$ and CKD has become increasingly noted.

\section{CHRONIC KIDNEY DISEASE}

\section{Epidemiology and Defining CKD}

CKD is defined as a gradual loss of kidney function accompanied by kidney damage, usually measured by albuminuria. CKD is typically accompanied by interstitial fibrosis, glomerulosclerosis, and chronic inflammation.[13] A diagnosis of CKD encompasses a diverse range of conditions with different causes, severity, and rates of progression. Typically, CKD is diagnosed when patients progress to the failure stage of the RIFLE classification and remain there for over 3 months.[15] At this stage there is generally a 75\% reduction in GFR. Since no treatment is available for CKD, patients must be put on dialysis. This can slow the progression of kidney disease, but patients will eventually progress to loss of kidney function (complete loss of function for more than 4 weeks) and then to end stage renal disease (complete loss of function for more than 3 months). Patients at this stage eventually require a renal transplant to survive.[5] 
CKD prevalence has been steadily increasing over the past 20 years.[16] As of 2017, CKD was estimated to affect $13.4 \%$ of people globally.[17] In the US, there is estimated to be 400 new cases of CKD per million people per year.[15] This increasing prevalence is concerning due to the detrimental impact on patients as well as the high societal cost of CKD. Generally, CKD development is associated with old age, hypertension, diabetes, cardiovascular disease, and obesity.[15] Recently, more studies have been done revealing that multiple incidences of AKI also increase the likelihood of CKD development.[18, 19]

As mentioned earlier, AKI involves high levels of tubule cell death. Following AKI, the kidney initiates normal wound healing processes to recover from the initial insult. Although the repair processes following AKI can recover renal function, in some cases the kidney undergoes what has been termed maladaptive repair, which can lead to the development of CKD.[13]

\section{Maladaptive Repair}

Maladaptive repair refers to the phenomenon by which normal repair processes go awry and result in progressive damage. The primary features of maladaptive repair are cell cycle arrest, profibrotic cytokine production, myofibroblast accumulation, chronic immune cell activation, and chronic vascular impairment.[13] Maladaptive repair begins in the maintenance phase of AKI, when tubule cells are beginning to respond to renal injury. In this stage, it has been demonstrated that surviving tubule cells are pushed into the cell cycle in order to proliferate and re-establish the proximal tubule structure.[20] This adaptive proliferation is necessary for healing, but in maladaptive repair tubule 
cells undergo G2/M cell-cycle arrest and senescence.[13] In this arrested state tubule cells secrete profibrotic factors such as transforming growth factor beta (TGF $\beta$ ) and connective tissue growth factor (CTGF) downstream of c-Jun Nterminal kinase (JNK) activation.[12]

The process of cell-cycle arrest and subsequent JNK activation leading to fibrosis has been primarily worked out in models of ischemia-reperfusion injury (IRI). Yang et. al. demonstrated that pharmacologic inhibition of JNK as well as p53 (preventing cell-cycle arrest) reduces fibrosis following ischemic injury. This study also found that pharmacologic induction of G2/M cell cycle arrest in HK-2 cells increased JNK activation and upregulated profibrotic gene expression.[21] These studies demonstrate that cell cycle arrest can lead to JNK activation and cytokine production. It also illustrates that this process is involved in development of renal fibrosis following ischemic injury.

The profibrotic cytokines produced by senesced tubule cells promotes production of activated myofibroblasts.[12] A major hallmark of maladaptive repair is the appearance of these activated myofibroblasts, which are the main contributors of collagen deposition and development of the fibrotic matrix.[13] Myofibroblasts display characteristics of both smooth muscle cells and fibroblasts. In normal wound healing their role is to deposit ECM in order to maintain the integrity or injured tissue, allowing for proliferation and healing. In maladaptive repair, myofibroblasts are chronically stimulated leading to over production of ECM. The ECM laid down in renal fibrosis is primarily composed of type I and III collagen cross-linked by fibronectin.[22] Myofibroblasts have been 
speculated to differentiate from several different precursors.[23] Although it is still a subject with much controversy, resident fibroblasts and pericytes are thought to be major contributors with mesenchymal stem cells and bone-marrow derived fibrocytes also having a role.[13]

In addition to profibrotic cytokine production, injured tubule cells also release pro-inflammatory cytokines and chemokines through mechanisms including activation of toll-like receptor (TLR) signaling. TLRs are expressed on tubule epithelial cells. Exogenous and endogenous ligands associated with injury can activate the proinflammatory TLR response leading to production of tumor necrosis factor alpha (TNFa), monocyte chemoattractant protein 1 (MCP-1), and interleukin 6 (IL-6) to recruit immune cells to the injured kidney.[12] Neutrophils, monocytes/macrophages, and leukocytes are among the most responsive infiltrates to the kidney. These immune cells are important in the normal process of recovery to attenuate early injury as well as clear damaged debris. However, chronic immune cell activation can mediate effects of maladaptive repair, particularly through chronically activated macrophages.[13]

Macrophages make up a large and diverse population of immune cells. They are a group of phagocytic cells that differentiate from monocytes upon environmental stimulation. In kidney injury, both renal resident and bone-marrow derived infiltrating macrophages respond to injury. Resident macrophages arise from embryonic macrophages that migrate to the kidney during development. These resident cells help maintain renal homeostasis by clearing cellular debris and aiding in tissue remodeling. Infiltrating macrophages are recruited to the 
kidney following injury. Both resident and infiltrating macrophages can adopt different phenotypes to perform a variety of functions.[24]

Functionally speaking, macrophages display a spectrum of subtypes. For simplicity, we will refer to the extreme ends of the spectrum as M1 and M2. M1 macrophages appear in the kidney rapidly following injury. These cells are generally considered "pro-inflammatory." Macrophages are polarized to an M1 phenotype by pro-inflammatory cytokines and danger signals.[25] Upon polarization, they continue to produce proinflammatory cytokines to recruit other immune cells. They also produce reactive oxygen and nitrogen species to destroy pathogenic particles. This is done largely by inducible nitric oxide synthase (iNOS) which is a characteristic marker of M1 function.[24] M1 macrophages are thought to play a role in infection clearance, but they are also known to exacerbate acute renal injury.[25]

M2 macrophages appear later in kidney injury and are thought of as having a "pro-repair" phenotype. There are several different subsets of M2 macrophages that differentiate in response to different environmental stimuli. TGF $\beta$ is one of the major cytokines that drives M2 polarization. M2 macrophages are thought to suppress inflammation and promote tissue repair.[25] Arginase-1 (Arg-1) is characteristically expressed by M2 macrophages and is used to produce building blocks for the ECM. M2 macrophages also secrete TGF $\beta$ creating a positive feedback signaling loop.[24] Although M1 macrophages may still be present in chronic injury, development of renal fibrosis is associated with an M1 to M2 phenotype transition.[25] 
The complex role of macrophages in kidney injury has been studied by several groups. In a model of IRI it was demonstrated that global depletion of macrophages via administration of liposome clodronate attenuated development of renal fibrosis. Adoptive transfer of M2 macrophages after global depletion reversed this beneficial effect while M1 transfer did not. This suggests a role for M2 macrophages in the development of fibrosis post-IRI.[26] Another study went on to demonstrate that global depletion of macrophages with liposomal clodronate after development of AKI following IRI resulted in failure to recover from injury.[27] These studies demonstrate the importance of coordinated M1 and $\mathrm{M} 2$ responses to kidney injury to promote proper healing and repair. Chronic activation of either subset likely can exacerbate maladaptive repair processes.

The immune cell responses and wound healing processes observed after a renal insult suggests repairing tubule structure is the most immediate response to kidney injury. However, removing causes of injury is also of high importance in recovery from $\mathrm{AKI}$. As mentioned earlier, vascular endothelial damage in AKI leads to renal hypoxia. In the extension phase of AKI, this endothelial injury and dysfunction leads to continued impairment of kidney oxygen delivery. Studies show repeated injury promoting tubule cell proliferation may impede the repair of endothelial cells. Tubule proliferation is associated with high levels of TGF $\beta$ and low levels of vascular endothelial growth factor (VEGF) in the kidney. Differentiation of pericytes into myofibroblasts trigged by damaged tubule cells also removes vital trophic support of endothelial cells. This suggests that tubular repair may inadvertently impair vasculature repair.[13] Repeated AKI insults and 
maladaptive repair therefore also lead to chronically impaired vasculature, further promoting renal fibrosis.

All the above processes demonstrate how maladaptive repair can result from a chronic activation of injury responses. Endothelial damage promotes continued injury leading to tubule G2/M cell-cycle arrest, profibrogenic cytokine production, chronic immune cell activation, and activation of myofibroblasts. These processes promote chronic inflammation, fibrosis, and vascular rarefaction characteristic of CKD.[13]

Fibrosis and Resolution

Fibrosis is defined as an excessive accumulation of ECM commonly caused by chronic or repeated injury. Excessive ECM deposition disrupts tissue architecture and decreases renal function.[22, 28] Typically, ECM production is a normal part of wound healing. It is vital to maintaining tissue integrity following an injury. Improper resolution of fibrosis is what leads to chronic disease. Wound healing can be defined in phases that involve the same processes as maladaptive repair up until the final phase. The inflammatory phase refers to the death of tubule cells prompting infiltration of immune cells to clear debris and promote new tissue formation.[22] The proliferative/effector phase involves the proliferation of tubule cells to reestablish barrier function as well as the differentiation and proliferation of myofibroblasts to produce ECM and maintain tissue integrity. The final phase of wound healing is maturation. In this stage, the ECM is degraded and profibrogenic myofibroblasts are removed. Repeated or 
chronic injury often leads to inadequate maturation, resulting in long term organ fibrosis.[28]

It should be noted that there are two main forms of ECM: basement membrane ECM and interstitial ECM. Basement membrane ECM provides support for epithelial and endothelial cells. It is made up of collagen IV and laminins. Interstitial ECM is secreted primarily by myofibroblasts and has a more rigid, cross-linked structure. Interstitial ECM is thought to be more fibrotic, made up of fibrillar collagen type I and III cross-linked by fibronectin. Basement membrane and interstitial ECM are thought to play different roles in the development of renal fibrosis.[22]

Resolution of organ fibrosis in the maturation phase requires three components: eradicating the cause of injury, degradation and removal of the fibrotic ECM, and elimination of myofibroblasts.[22, 28] Promoting resolution of organ fibrosis to treat CKD is a rising area of research. One mechanism for targeting resolution of fibrosis is increasing the degradation of ECM. A potential mechanism for increasing the degradation of ECM is through metalloproteinases (MMPs). MMPs are thought to be the most important enzymes involved in collagen degradation. They comprise a large family of proteins. Although MMPs are important in degradation of fibrotic matrix, they can also promote fibrosis through activities unrelated to ECM degradation.[22]

The role of MMPs in renal fibrosis is not straight forward. Nishida et. al. examined the role of matrix metalloproteinase 2 (MMP2) in development of renal fibrosis following unilateral ureteral obstruction. They found that pharmacologic 
inhibition of MMP2 attenuated development of fibrosis. Interestingly, MMP2 inhibition also lead to decreased macrophage infiltration in the injured kidney. This was hypothesized to be the mechanism of protection.[29]

Wang et. al. also demonstrated that mice lacking matrix metalloproteinase-9 (MMP9) developed less fibrosis in a model of unilateral ureteral obstruction. MMP9 knockout mice displayed less disruption of tubule basement membrane following injury. They also had decreased expression of fibronectin and less total collagen. They hypothesized that MMP9 deficiency provided protection primarily by preventing basement membrane degradation.[30] Other studies on MMP9 have led to the hypothesis that MMP9 degradation of basement membrane ECM promotes tubule epithelial to myofibroblast transition. Promoting this differentiation suggests that MMP9 plays a profibrogenic role.[31, 32]

Other studies have also demonstrated the complexity of MMPs in renal fibrosis. Another strategy that has been tried to promote fibrotic ECM degradation is inhibition of tissue inhibitors of metalloproteinases (TIMPs). TIMPs bind to MMPs and target them for macrophage engulfment, preventing their activity.[22] Kawamoto et. al. used TIMP3 knockout mice in a model of unilateral ureteral obstruction. Although these mice had some alterations in kidney structure, TIMP3 deficiency did not prevent development of renal fibrosis.[33]

These studies indicate that MMPs and TIMPs play multiple roles in the kidney besides regulating degradation of fibrotic ECM. The balance of MMPs and TIMPs can regulate immune cell responses, myofibroblast accumulation, and 
signaling pathways such as TGF $\beta$. It is clear that MMPs and TIMPs serve purposes beyond degradation of ECM. Our lack of understanding of their many complex roles make them a difficult target for prevention of renal fibrosis.[28]

As myofibroblasts are thought to be the source of increased ECM in development of fibrosis, eliminating myofibroblasts is another area of focus. Myofibroblasts may be removed via apoptosis, senescence, dedifferentiation, or reprogramming.[22] Zeisberg et. al. attempted to promote myofibroblast dedifferentiation to attenuate renal fibrosis in a nephrotoxic serum nephritis model. This group used bone morphogenic protein 7 (BMP-7), a member of the TGF $\beta$ superfamily, in an attempt to reverse the epithelial to myofibroblast transition occurring in progressive renal disease. In vitro they demonstrated that BMP-7 reversed TGF $\beta$-induced changes in tubule epithelial cells. They therefore hypothesized that BMP-7 was promoting processes that opposed the epithelial to myofibroblast transition and would be capable of preventing myofibroblast accumulation. They then demonstrated that BMP-7 treatment in vivo slowed progression of renal fibrosis, but it could not reverse disease pathogenesis.[34] Although this study showed promise, the plasticity of myofibroblasts make them a difficult target for treatment.

Although fibrosis shows potential for resolution in the context of wound healing, it is accepted that all organs have a "point of no return" in which capacity for resolution is lost. In most clinical cases, the attempts to reverse developed organ fibrosis have not had much success.[28] Targeting the extension phase of AKI seems to hold the most potential for preventing the AKI to CKD transition. 
Currently, there is no cure for CKD. As CKD is a progressive disease, most therapies are aimed at supportive care and slowing the progression of functional decline. Medication aimed at blood pressure and glycemic control are commonly used.[35] Even with these treatments patients will eventually progress to endstage renal disease.

\section{CISPLATIN}

\section{Clinical Uses of Cisplatin}

As previously mentioned, the most common cause of intrinsic $\mathrm{AKI}$ is acute tubular necrosis caused by ischemic or nephrotoxic insults. Nephrotoxic side effects of drugs cause around $20 \%$ of hospital and community acquired AKI cases.[36] One of the most common class of agents that induce nephrotoxic acute tubular necrosis is nephrotoxic chemotherapies.[11] It is estimated that $60 \%$ of hospital-acquired $\mathrm{AKI}$ is caused by nephrotoxicity of chemotherapy.[37]

Cisplatin (cis-diamminedichloridoplatinum(II)) is an inorganic platinumbased drug that was approved by the FDA as an anticancer agent in 1978.[38] It is currently a standard component of therapy used to treat many solid-organ cancers such as head, neck, lung, testis, ovary, and breast.[39] The major mechanism of action of cisplatin involves binding DNA, forming adducts, and forcing cancer cells to undergo apoptosis or cell-cycle arrest. It has shown a great amount of success as an anticancer agent, particularly in treating testicular and ovarian cancer. Since the FDA approval of cisplatin, the rate of remission for 
testicular cancer has increased from $5 \%$ to around $80 \%$.[38] The effectiveness of cisplatin however, is largely reduced by its dose-limiting nephrotoxicity.

In an effort to bypass this toxicity, several analogs of cisplatin such as carboplatin and oxaliplatin have been developed. Although these compounds do have less nephrotoxicity, they are much less effective in killing many types of cancer cells. One study demonstrated that the concentration of carboplatin had to be $20-40$ fold higher than that of cisplatin to form the same amount of DNA adducts.[38] Due to its high level of activity, cisplatin is still commonly used in the clinic today.

Around $30 \%$ of patients who receive cisplatin develop AKI.[39] Since there are no approved agents to treat $\mathrm{AKI}$, development of the injury requires treatment to be suspended. Patients are monitored in hopes that renal function will recover in 2-4 weeks. Following recovery, patients are often switched to a different treatment regimen.[39] The nephrotoxicity associated with cisplatin prevents it from being as effective in treating cancer as it could be. Since its approval, research has shed light on the mechanism of cisplatin's nephrotoxicity in hopes of finding a way to prevent these effects.

\section{Mechanisms of Nephrotoxicity}

Patients typically receive cisplatin as either a bolus intravenous injection or slow intravenous infusion.[40] As the kidney receives a large portion of blood flow, it encounters high concentrations of cisplatin. Cisplatin is filtered from the blood into the kidney through both glomerular filtration and tubular secretion. As 
the blood is filtered by the kidney, active accumulation of cisplatin within renal parenchymal cells occurs. Cisplatin concentrations eventually become greater in the kidney then in the blood.[39]

The high affinity copper transporter (Ctr1) and the organic cation transporter (OCT2) are both highly expressed basolaterally on proximal tubule cells and facilitate the uptake of cisplatin by the kidney.[41] While Ctr1 is ubiquitously expressed, OCT2 has much higher levels of renal expression.[37] Ctr1 has been shown to facilitate uptake of cisplatin by cancer cells, making it a poor target for prevention of renal toxicity.[41] OCT2 expression is more specific to renal tubule cells and studies have demonstrated that OCT2 knockout and pharmacologic inhibition with cimetidine confers some resistance to cisplatininduced kidney injury.[42, 43] Additional studies also demonstrated that coadministration of cisplatin with cimetidine did not affect antitumor efficacy.[44] On the apical membrane, multidrug and toxin extrusion 1 (MATE1) is expressed and is capable of transporting cisplatin from tubule cells into the lumen for excretion. Studies have shown that MATE1 knockout mice are more sensitive to AKI and have elevated levels of platinum in the kidney following high-dose cisplatin treatment.[45]

Prior to transport, metabolism of cisplatin begins in circulation with glutathione conjugation, possibly by glutathione-S-transferase.[39, 41] These conjugates are then cleaved by gamma glutamyl transpeptidase (GGT) to form cysteinyl-glycine-conjugates.[39] Some studies have demonstrated the inhibition of glutathione-S-transferase and GGT can attenuate cisplatin nephrotoxicity in 
mice, but the effect on tumoricidal activity is unknown.[37] Aminopeptidases further metabolize cisplatin by cleaving it into a cysteine conjugate. The cysteine conjugate is then transported into proximal tubule cells where it is converted to a highly reactive thiol by cysteine-S-conjugate-beta-lyase.[37, 39]

As mentioned above, the primary mechanism of cisplatin's anti-cancer activity is thought to be through DNA damage. Several studies have further demonstrated that mitochondrial DNA is the major target of damage.[39] In aqueous environments cisplatin exchanges its chloride ions for water molecules, forming a positively charged electrophile. This can lead to preferential accumulation in negatively charged mitochondria.[39, 46] Nevertheless, accumulation of reactive cisplatin metabolites in the cell leads to formation of DNA, RNA, and protein adducts.[47] These adducts contribute to nephrotoxicity via many of the same mechanisms by which cisplatin kills cancer cells. Cisplatininduced nephrotoxicity involves proximal tubule cell damage, increased oxidative stress, inflammatory response, and vascular injury.[41, 47]

Cisplatin induces proximal tubule cell damage via several mechanisms. The most prominent mechanism of injury is activation of cell death pathways.[39, 41, 47] Cisplatin has been demonstrated to induce necrosis as well as intrinsic-, extrinsic-, and endoplasmic reticulum (ER) stress associated pathways of apoptosis.[41, 47] Wei, Dong, \& Dong demonstrated the importance of intrinsicapoptotic signaling in cisplatin nephrotoxicity using genetic manipulation of proapoptotic BCL-associated X (BAX). They demonstrated that BAX knockout conferred resistance to cisplatin-induced kidney injury in mice by decreasing 
cytochrome $c$ release and the number of apoptotic cells. [48] This study indicates that tubule cell intrinsic apoptosis is a large contributor to nephrotoxicity.

Wei et. al. went on to demonstrate a role for tumor protein 53 (p53) in intrinsic apoptosis induction in cisplatin nephrotoxicity. p53 has been known to play a role in apoptotic induction following DNA damage. Their group observed that cisplatin treatment lead to increased phosphorylation of p53. They then showed that inhibition of p53 with pifithrin- $\alpha$ and genetic deletion of p53 attenuated cisplatin-induced kidney injury in mice.[49] This study demonstrates that DNA damage is likely a major contributor to intrinsic apoptotic induction in cisplatin nephrotoxicity.

Cisplatin has also been reported to promote cell death independently of its DNA damaging actions. Mandic et. al. observed nucleus-independent apoptotic signaling triggered by cisplatin. When enucleated cells were treated with cisplatin, ER stress was induced. This ER stress then triggered caspase activation and apoptosis. Other DNA damaging agents were not able to induce this same response, indicating that cisplatin had unique cytosolic targets.[50] Cisplatin-induced tubular damage sets off the pathological processes of AKI discussed earlier.

Cisplatin induces many other forms of sublethal damage involving cell cycle dysregulation, activation of mitogen activated protein kinase (MAPK) signaling, mitochondrial dysfunction, ER stress, and autophagy induction.[39, 41, 47] ER stress and autophagy are of particular relevance to this work. ER stress is caused by an accumulation of misfolded proteins. Cisplatin has been speculated 
to cause ER stress through adduction to cytosolic targets.[50] The level and duration of ER stress determines if cells are forced to undergo apoptosis or to adapt and survive. One method of adapting to ER stress is through upregulation of autophagy.[51] Autophagy is a form of cellular "self-eating" that can be both protective and detrimental. Studies have shown that cisplatin-induced AKI is associated with induction of autophagy. In acute models of injury this induction is thought to be protective.[47] Autophagy can help reduce levels of cisplatininduced apoptosis by ameliorating effects of cellular damage from oxidative stress and mitochondrial dysfunction.[52] In chronic kidney injury the role of autophagy is more controversial. It has been argued that long-term upregulation of autophagy could also contribute to renal damage.[53] These mechanisms of sublethal damage demonstrate that even if cisplatin does not induce cell death, it can still have negative impacts on cellular homeostasis.

Inflammation is another mediator of cisplatin nephrotoxicity that has been repeatedly studied in cisplatin-induced kidney injury (CDDP-KI). Cisplatin treatment is associated with an upregulation of many different proinflammatory cytokines and chemokines such as interleukin 1 beta (IL-1 $\beta$ ), IL-6, MCP-1, and TNFa. $[41,47]$ One mechanism of production of these signals is via toll-like receptor signaling. Injury to renal epithelial cells induces release of damage associated molecular patterns (DAMPs) which activate toll-like receptor 4 (TLR4). TLR4 activation leads to production of chemokines and cytokines, including TNFa, that lead to upregulation of endothelial adhesion molecules and attraction of inflammatory cells.[39] Ramesh and Reeves have shown that TNFa 
is a major inflammatory mediator in CDDP-KI. They utilized TNFa knockout mice and GM6001, a pharmacological inhibitor of TNFa, to demonstrate that inhibition of TNFa signaling attenuated CDDP-KI. They also demonstrated that TNFa inhibition decreased production of other inflammatory mediators, indicating it plays a central role in activation of the cytokine response.[54] The cytokine response attracts immune cell infiltrates to the injured kidney. Neutrophils, macrophages, T cells, and dendritic cells appear to be the largest responders to cisplatin induced injury.[39, 47]

Vascular damage is another mediator of CDDP-KI. This damage is orchestrated in a variety of ways including alteration of renal blood flow and direct toxicity to endothelial cells.[47] Early vasoconstriction in response to cisplatin has been thought to be an initiator of vascular injury. Although the cause of vasoconstriction is not quite clear, it results in a decrease in GFR and promotes development of a hypoxic environment. Hypoxic conditions further contribute to the tubule cell death and inflammation. Vascular damage, tubule cell death, and inflammation are all connected and work together to orchestrate the nephrotoxicity of cisplatin.[4]

The nephrotoxicity of cisplatin has been observed in patients since its introduction. Many strategies have been applied to prevent this toxicity from occurring. Hydration regimens and use of diuretics such as furosemide and mannitol have been explored. Clinically, however, these regimens do not seem to prevent CDDP-KI. Current clinical guidelines recommend pre-hydration with $0.9 \%$ saline and avoidance of diuretics.[39] 


\section{Models of Cisplatin Injury}

As previously mentioned, $30 \%$ of patients who receive cisplatin develop AKI. They display a rapid decline in kidney function and increase in BUN or serum creatinine following treatment. This type of CDDP-KI is modeled in animal studies by using a single high dose $(10-30 \mathrm{mg} / \mathrm{kg})$ of cisplatin. Following treatment, mice display a significant decline in renal function and typically only survive 3-4 days post treatment. This model mirrors the rapid decrease in GFR and spike in serum creatinine defining AKI in the clinic. However, it does not allow for long term studies of the effects of CDDP-KI.[38, 55]

$70 \%$ of patients who receive cisplatin do not develop AKI by clinical criteria. Recent studies have revealed that these patients may still be at risk for long term renal effects from cisplatin. Skinner et. al. followed pediatric patients treated with cisplatin that did not develop clinical AKI. They found that 10 years following treatment $11 \%$ had a decreased GFR and $15 \%$ were showing signs of nephrotoxicity.[56]

In order to address this issue, Sharp et. al. developed a new animal model to study CDDP-KI. This model involves repeated low doses of cisplatin (7-9 $\mathrm{mg} / \mathrm{kg}$ ) once a week for four weeks. Mice treated with this dosing regimen can survive 6 months following treatment. Immediately following treatment mice do not show significant increases in BUN; however, at 6 months post-treatment mice do have significantly elevated BUN levels compared to vehicle treated mice. This indicates mice have progressive loss of kidney function following cisplatin treatment without showing clinical signs of AKI during the dosing window.[57] 
These models together allow us to study patients on either end of the CDDP-KI spectrum. The acute, high dose model is characterized by high levels of tubular apoptosis and necrosis, a rapid decline in GFR, and increases in BUN and serum creatinine. On the other hand, the chronic, low dose model does not cause significant BUN or serum creatinine changes. This model seems to cause subclinical injury that leads to development of fibrosis following the fourth dose. Fibrosis worsens as mice age to 6 months post treatment, accompanied by a chronic infiltration of macrophages. This phenotype is more indicative of CKD development.[58]

Together these models demonstrate the interconnectedness of $\mathrm{AKI}$ and CKD and how cisplatin can lead to both types of kidney injury. These models also depict how different biological processes can be triggered in response to acute or chronic cisplatin treatment. Both models should therefore be used to test drug candidates to prevent CDDP-KI.

\section{SPHINGOLIPIDS AND KIDNEY INJURY}

\section{Sphingolipid Metabolism}

Sphingolipids are lipid compounds that contain a sphingoid-base backbone. The kidney has been known to play an important role in sphingolipid metabolism for many years. It was originally thought that sphingolipids played primarily a structural role in the cell. It has since come to light that sphingolipids act on a number of cellular targets and are involved in many different signaling 
pathways.[59] It has become an increasing area of interest to examine how these bioactive lipids effect renal physiology and how regulation of lipid levels could be used as a therapeutic target.[60]

Sphingolipid metabolism involves an abundance of enzyme-catalyzed reactions. At the center of this large network lies ceramide. Ceramide can be produced via de novo synthesis, sphingomyelin hydrolysis, or recycling of other complex sphingolipids. In de novo synthesis, serine palmitoyl transferase condenses serine and palmitoyl CoA to form 3-ketosphingosine. This product is then reduced to dihydrosphingosine by 3 -ketoreductase. Dihydrosphingosine is then acylated by ceramide synthase to form dihydroceramide. Desaturase then desaturates dihydroceramide at the 4-5 position of the sphingoid base backbone to generate ceramide. Once generated, ceramide is the basis for several reactions generating other bioactive lipid species.[59, 61]

Ceramide can be used by sphingomyelin synthases to form sphingomyelin. It can also be phosphorylated by ceramide kinase to form ceramide-1-phosphate. Glycosylation of ceramide via addition of a glucose or galactose forms the hexosylceramides, glucosylceramide, and galactosylceramide, respectively. Hexosylceramides are utilized to synthesize more complex gylcosphingolipids and can also be broken down to regenerate ceramide. Ceramide can be cleaved via the action of ceramidases to form sphingosine, which can then be phosphorylated by sphingosine kinases 1 or 2 to form sphingosine-1-phosphate (S1P). Sphingosine can also be recycled back into ceramide as catalyzed by ceramide synthases or ceramidases. In our 
studies, we focus on the balance of ceramide, sphingosine, and S1P. It is thought that the balance of these bioactive sphingolipids regulates several cellular processes including inflammation, reactive oxygen species production, and cell death. These are all processes implicated development of acute kidney injury and chronic kidney disease.[59, 61]

Manipulation of Sphingolipids in Models of Kidney Injury

Ceramide has been considered a "pro-apoptotic" signaling lipid in the past.[59] In vitro, cisplatin treatment of baby mouse kidney epithelial cells induces ceramide generation via upregulation of de novo ceramide synthesis. Inhibition of ceramide synthesis protected these cells from cisplatin-induced cell death.[61] Our studies have also demonstrated that acute cisplatin treatment causes increased levels of ceramide in the kidney.[62] Taken together, these studies suggest that ceramide could be a key mediator of cisplatin-induced cell death in kidney injury.

Dupre et. al. used this rationale to explore the impact of inhibiting ceramide synthesis on kidney injury caused by acute cisplatin treatment. In this study, it was also observed that there were increased activities of ceramide synthases in the de novo synthesis pathway and acid sphingomyelinase in the kidney cortex following treatment of mice with cisplatin. Therefore, ceramide synthesis was inhibited via myriocin and amitriptyline, inhibitors of de novo ceramide synthesis and acid sphingomyelinase, respectively. These inhibitors reduced ceramide levels in the kidney following cisplatin treatment. This inhibition 
also resulted in lower levels of BUN and serum creatinine following single, high dose cisplatin.[62]

Dupre et. al. also noted that there were increased levels of hexosylceramides in the kidney following cisplatin treatment of mice. It had been unknown whether ceramide or the shuttling of ceramide into hexosylceramides was promoting CDDP-KI. C10, an inhibitor of glucosylceramide synthase, was used to prevent this shuttling of ceramide into hexosylceramides. C10 and cisplatin co-treatment of mice lead to increased levels of BUN, Kim-1, and tissue damage. C10 also caused increased apoptosis, cell proliferation, and inflammatory cytokine production following cisplatin treatment. C10 and cisplatin co-treatment prevented hexosylceramide generation, but increased ceramide accumulation.[62] These data suggest a role for ceramide in apoptosis in CDDP$\mathrm{KI}$. It also provides an example of how regulating the balance of different sphingolipid species can affect CDDP-KI.

Other groups have also explored the role of sphingolipids in acute kidney injury. The Okusa lab explored the role of S1P in kidney injury. S1P is known to bind to and signal through a family of G-coupled protein receptors known as S1P receptors (S1PRs). S1PR signaling was shown to be protective in models of acute cisplatin injury and IRI. A S1PR agonist offered protection from kidney injury in these models while genetic S1PR deletion exacerbated injury.[63, 64] Bartles et. al. also demonstrated the importance of SIP signaling in IRI. They subjected mice with endothelial specific S1PR deletion to ischemic injury. These mice sustained higher levels of injury then wild type controls. This study 
highlights the importance of S1PR in endothelial function.[65] These studies demonstrate the importance of S1P signaling pathways in renal injury.

The homeostasis of ceramide and other sphingolipids in the context of kidney injury is becoming increasingly recognized. In this study, we evaluate the effects of neutral ceramidase inhibition in the context of kidney injury both in vitro and in vivo. Neutral ceramidase (nCDase) is one of five ceramidases. It is named because it is most active at neutral pHs.[66] Although the role of nCDase in kidney injury has not received much attention, it has been identified as a target in other models of injury. Novgorodov et. al. demonstrated that nCDase inhibition conferred protection from traumatic brain injury by protecting cells from mitochondrial damage.[67] In the kidney, nCDase activity was shown to be elevated in models of diabetic neuropathy.[68] Taken together, these studies indicate that nCDase could play a pathological role in kidney injury via alteration of ceramide, sphingosine, and S1P balance.

Our lab demonstrated that mouse embryonic fibroblasts (MEFs) lacking functional nCDase were protected from nutrient and energy deprivation induced cell death. nCDase knockout cells could survive cell stress due to increased levels of autophagy.[69] It has been demonstrated that autophagy plays a protective role in acute cisplatin-induced renal injury.[70] We therefore hypothesized that nCDase inhibition may have some effect on CDDP-KI development.

With the growing body of knowledge delineating the AKI to CKD transition it has become increasingly important to test prospective nephroprotective targets 
in models of both acute injury and fibrosis development. In this study we set out to examine how nCDase inhibition affects kidney injury following both the acute high-dose cisplatin model and the repeated low dose cisplatin (RLDC) model. Our results indicate that repeated low dose cisplatin treatment may utilize unique mechanisms of injury to promote CKD development. 


\section{MATERIALS AND METHODS}

\section{Cell Culture}

Mouse embryonic fibroblast (MEFs) were generated and validated as previously described from either wild type or neutral ceramidase (nCDase)-null C57BL/6 mice that were littermates.[69] Cells were immortalized with dominant-negative p53. MEFs were maintained in Dulbecco's modified Eagle's medium (DMEM) containing $10 \%$ fetal bovine serum (FBS) and supplemented with L-glutamine, penicillin, streptomycin, and ciprofloxacin (Invitrogen). All cells were incubated at $37^{\circ} \mathrm{C}$ in $5 \%$ carbon dioxide. Cells were not cultured for more than 30 doublings and were routinely assessed for mycoplasma using MycoSensor PCR assay kit (\#302108, Agilent Technologies) according to the manufacturer's protocol.

\section{Cell Viability Assays}

For the FBS withdrawal experiment, cells were plated in DMEM containing $10 \%$ FBS and antibiotics into 3 separated 96 -well plates on day 1 . On day 2 , cells were washed with sterile phosphate buffered saline (PBS) and then treated with media at varying concentrations of FBS. On day 3, 1 plate was assessed for 24hour viability using Alamar Blue assay. On day 4, the 48-hour plate was assessed and on day 5, the 72-hour plate was assessed. For the nCDase inhibitor viability assay cells were plated, treated, and assessed in the same manner. C6-urea-ceramide (C6) and the nCDase inhibitor from Ono 
Pharmaceutical Company (Ono) were dissolved in dimethyl sulfoxide (DMSO) and added to DMEM at indicated concentrations. Final DMSO concentration in media was kept under $1 \%$.

\section{nCDase in vitro TLC Activity Assay}

Equal amounts of recombinant nCDase (gifted from Dr. Yusef Hannun, Stony Brook University) were incubated and treated with the indicated nCDase inhibitors at $0.1 \mu \mathrm{M}$ to $100 \mu \mathrm{M}$. NBD-ceramide at $50 \mu \mathrm{M}$ in $100 \mathrm{mM}$ Tris with $0.3 \%$ Triton-X-100, $\mathrm{pH} 7.5$ was added to each sample. The reaction progressed for 10 minutes and was then stopped with 3:1 addition of equal parts $\mathrm{MeOH}$ :Chloroform. Samples were centrifuged at 15,000 rpm for 10 minutes at $4^{\circ} \mathrm{C}$. The organic phase of the reaction was extracted and dried down in a speed vacuum. Samples were then solubilized in $10 \mu \mathrm{L}$ of 1:1 MeOH:Chloroform and spotted on a TLC plate. The plate was placed in a reaction chamber with 90:29.5:0.5 Chloroform:MeOH: $\mathrm{NH}_{4} \mathrm{OH}$ for 1 hour to allow separation of ceramide and sphingosine. Plates were imaged with phosphoimager and analyzed using Quantity One software. This assay was modified from Tani et. al.[71]

\section{Animals}

Neutral ceramidase knockout mice $\left(\mathrm{nCDase}^{-/}\right)$were gifted from Dr. Ashley Snider at Stony Brook University. nCDase ${ }^{-/-}$were originally generated in the laboratory of Dr. Richard L. Proia (NIDDK, National Institutes of Health). These mice were previously backcrossed for 10 generations onto a C57BL/6 background. $\mathrm{nCDase} \mathrm{e}^{-/}$mice were bred in-house and all experiments were 
performed at 8-10 weeks of age. Wild type mice (C57BL/6, male, 4-weeks old) were either bred in house or purchased from The Jackson Laboratory and allowed to acclimate for 4 weeks prior to initiation of experiments. All mice were maintained on a 12-hour light/dark cycle and provided food and water ad libitum. All animal procedures were approved by the Institutional Animal Care and Use Committee and followed the guidelines of the American Veterinary Medical Association.

\section{Cisplatin Dosing Regimen}

Pharmacy grade cisplatin $(1 \mathrm{mg} / \mathrm{mL}$ in normal saline) was obtained from the University of Louisville Hospital pharmacy. For the high-dose study, cisplatin was administered at $20 \mathrm{mg} / \mathrm{kg}$ in normal saline in a total volume of $500 \mu \mathrm{L}$ by intraperitoneal (i.p.) injection. Vehicle treated mice received $500 \mu \mathrm{L}$ normal saline by i.p. injection. For the repeated low dose study, cisplatin was administered at 9 $\mathrm{mg} / \mathrm{kg}$ in normal saline in a total volume of $400 \mu \mathrm{L}$ by i.p. injection once a week for four weeks. Vehicle treated mice received $400 \mu \mathrm{L}$ normal saline by i.p. injection once a week for four weeks. All mice were administered $500 \mu \mathrm{L}$ saline by subcutaneous injection 1 day prior to dose 3,2 days after dose 3 , and 1 day before dose 4 to prevent weight loss. All animals were injected with cisplatin at the same time of day as there are circadian influences on the response of the kidney to cisplatin. Animals were euthanized 72 hours following their final cisplatin injection. Upon euthanasia, blood was collected and plasma prepared and frozen at $-80^{\circ} \mathrm{C}$; urine was collected and frozen at $-80^{\circ} \mathrm{C}$; and kidneys were removed, flash frozen in liquid nitrogen, and stored at $-80^{\circ} \mathrm{C}$ until use. A cross- 
section of one kidney was also fixed in $10 \%$ neutral buffered formalin for histology.

\section{Blood Urea Nitrogen (BUN), Serum Creatinine (SCr), and Neutrophil Gelatinase Associated Lipocalin (NGAL) Determination}

BUN (AMS Diagnostics, 80146) and SCr (Point Scientific Inc., C7548-120) levels were measured from plasma samples using the indicated kits following the manufactures' instructions. ELISAs for NGAL (R\&D Systems, DY1857) were performed on the urine as directed by the manufacturer.

\section{Protein Quantification and Western Blot Analysis}

Kidney tissue and cultured MEF cell pellets were homogenized in cell extraction buffer (Thermo Fisher Scientific) containing a Complete Protease Inhibitor Cocktail Tablet and Phosphatase Inhibitor Cocktail Tablets (Roche).

Homogenates were centrifuged at $15,000 \mathrm{Xg}$ for $10 \mathrm{~min}$ at $4^{\circ} \mathrm{C}$. Supernatants were removed and stored at $-80^{\circ} \mathrm{C}$. Protein concentrations were determined using Bradford Reagent (Bio-Rad). $40 \mu \mathrm{g}$ of kidney homogenate protein and 20 $\mu \mathrm{g}$ of MEF cellular protein were loaded and separated on $4-12 \%$ gradient TrisGlycine-SDS polyacrylamide gels. Protein was then transferred to PVDF membranes that were blocked in $5 \%(\mathrm{w} / \mathrm{v})$ dried milk in tris buffered saline $0.1 \%$ Tween 20 (TBST) for 1 hour. Membranes were incubated with primary antibody overnight at $4^{\circ} \mathrm{C}$. The next morning, membranes were washed 3 times for 5 min each with TBST containing 5\% (w/v) dried milk. Membranes were then incubated for 2 hours at room temperature with secondary antibodies conjugated with horseradish peroxidase $(1: 20,000)$ in TBST containing $1.25 \%(\mathrm{w} / \mathrm{v})$ dried milk. 
Following 3 washes in TBST membrane proteins were detected by chemiluminiscence substrate.

\section{Antibodies}

The following antibodies were purchased from Cell Signaling (Beverly, MA): cleaved caspase-3 (CC3) \#9664, C/EEBP homologous protein (CHOP) \#2895, phosphorylated c-jun n-terminal kinase (p-JNK T183/Y185) \#4668, c-jun nterminal kinase (JNK) \#9258 and \#9252, phosphorylated-protein kinase B (p-AKT S473) \#4060, protein kinase B (AKT) \#4691, phosphorylated-glycogen synthase kinase beta ( $p$-GSK3 $\beta$ S9) \#9322, glycogen synthase kinase beta (GSK3 $\beta$ ) \#9315, phosphorylated nuclear factor kappa-light-chain-enhancer of activated B cells (p-NF-KB S536) \#3033, nuclear factor kappa-light-chain-enhancer of activated B cells (NF-kB) \#3987, phosphorylated- eukaryotic initiation factor 2 alpha (p-elF2 $\alpha$ ) \#3398, and light chain 3B (LC3B) \#3868. Remaining antibodies include: fibronectin (F3648, Sigma-Aldrich), a-tubulin (SC-23948, Santa Cruz Biotechnology), and $\beta$-actin (A2228, Sigma-Aldrich).

\section{Gene Expression}

RNA was isolated using either TRIzol (Thermo Fisher Scientific) or E.Z.N.A. Total RNA Kit 1 (OMEGA) per manufacturer's protocol. cDNA was synthesized with High Capacity cDNA Reverse Transcriptase PCR (Thermo Fisher Scientific) per manufacturer's instructions. Gene-specific cDNA was quantified with real-time qRT-PCR using either predesigned TAQman assays or self-designed SYBR assays. The following TAQman primers were purchased from Thermo Fisher 
Scientific: tumor necrosis factor alpha (TNF- $\alpha$, Mm00443258_m1), interleukin-6 (IL6, Mm00446190_m1), chemokine (C-X-C Motif) ligand 1 (CXCL1, Mm04207460_m1), monocyte chemotactic protein (MCP-1, Mm00441242_m1), arginase-1 (Arg-1, Mm00475991_m1), and the housekeeping gene beta-2microglobulin (B2M, Mm00437762_m1). The following primers were selfdesigned: inducible nitric oxide synthase (iNOS, Invitrogen, forward:

GAGATTGGAGGCCTTGTGTCA, reverse: TCAAGCACCTCCAGGAACGT) and kidney injury molecule-1 (Kim-1, Invitrogen, forward:

AGATCCACACATGTACCAACATCAA, reverse:

CAGTGCCATTCCAGTCTGGTTT). qRT-PCR was done with either iTaq Universal Probes Supermix (172-5134, Bio-Rad) or iTaq Universal SYBR Green Supermix (172-5124, Bio-Rad).

\section{Histology}

Following formalin fixation, kidney tissue was processed and embedded in paraffin. Kidney sections $(5 \mu \mathrm{m})$ were stained with hematoxylin and eosin $(\mathrm{H} \& \mathrm{E})$ and periodic acid schiff (PAS). The degree of morphologic changes was determined by light microscopy in a blinded fashion. The following measures were chosen as an indication of morphologic damage to the kidney after drug treatment: proximal tubule degradation, loss of brush border, tubular casts, proximal tubule dilation, proximal tubule necrosis, presence of inflammatory cells, and interstitial fibrosis. These measures were evaluated on a scale from 0 to 4 , which ranged from not present (0), mild (1), moderate (2), severe (3), and very severe (4). 


\section{Sirius Red/ Fast Green Staining}

Kidney sections ( $5 \mu \mathrm{m}$ thick) were rehydrated in Histoclear followed by an ethanol gradient. Slides were then dipped in PBS with $0.1 \%$ Tween 20 and incubated for 5 minutes. Slides were washed with distilled water twice for 5 minutes each and then incubated in 1.2\% (w/v) saturated picric acid (\#5860-32, Ricca Chemicals) containing 0.1\% sirius red/direct red 80 (\#365548, Sigma) and $0.1 \%$ fast green FCF (\#F7258, Sigma). Slides were then washed with 5\% glacial acetic water until the water ran clear. Tissue samples were then dehydrated and fixed using Permount (\#17986-01, Electron Microscopy Sciences).

\section{Statistical Analysis Data}

Data are expressed as means \pm SEM for all experiments. Multiple comparisons of normally distributed continuous data were analyzed by two-way ANOVA and group means were compared using Tukey post-tests. Nonparametric continuous data were analyzed with a Kruskal-Wallis test followed by a Dunn's multiple comparison test. Nominal data from pathology scoring were analyzed by individual Chi-squared tests. The criterion for statistical differences was $p<0.05$ for all comparisons. 


\section{RESULTS}

nCDase knockout confers resistance to serum withdrawal in vitro. We had previously shown that nCDase knockout conferred resistance to ER stress- and nutrient deprivation- induced cell death.[69] These experiments were done with pharmacological agents that blocked cellular metabolism and/or directly acted on cellular organelles. To follow up these experiments, we also wanted to test this idea in a more natural model of ischemic injury. We used mouse embryonic fibroblasts (MEFs) grown from wild type and nCDase knockout C57BL/6 mice as described previously.[69] These cells were cultured in media at varying concentrations of fetal bovine serum (FBS) for 24, 48, and 72 hours. Cellular viability was monitored via measuring relative cell numbers through Alamar Blue fluorescence. We found that nCDase knockout cells had significantly higher viability at $0.625 \%$ and $0 \% \mathrm{FBS}$ at all timepoints compared to wild type controls. Viability was also significantly higher at $1.25 \%$ FBS at 48 hours (Fig 1). These data indicate that nCDase knockout provides protection from a serum withdrawal model of nutrient depletion. 


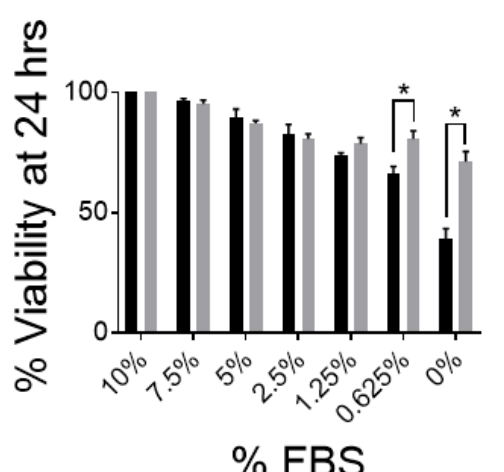

$\%$ FBS

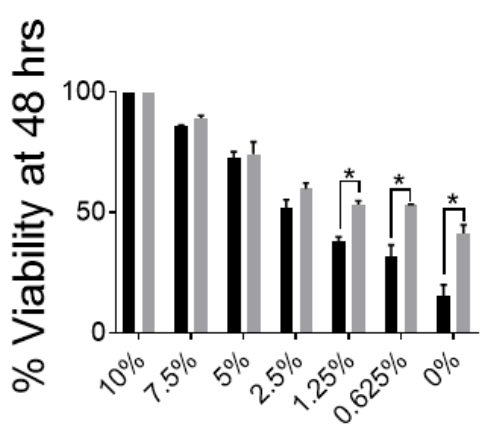

$\%$ FBS

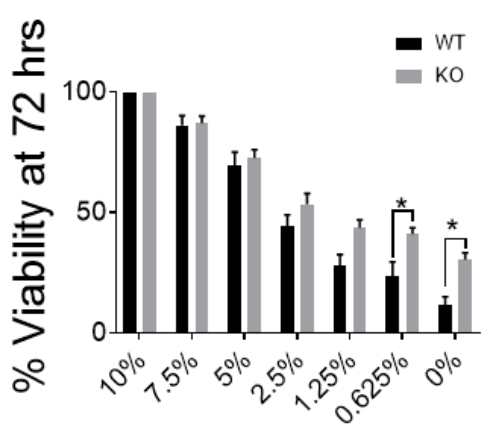

$\%$ FBS

Figure 1. Viability of nCDase knockout and wild type MEFs during FBS withdrawal.

Cells were plated at 2,000 cells per well in a 96-well plate on day 1 . The next day cells were washed with PBS then treated with the appropriate media. Viability was measured 24, 48 , and 72 hours from treatment via alamar blue assay. ${ }^{*}$ denotes significance of $p<0.05$. WT indicates wild type MEFs, KO indicates nCDase knockout MEFs. 


\section{nCDase knockout alters signaling pathways connected to autophagy and}

cell survival. It had previously been shown that upregulation of autophagy was the mechanism by which nCDase knockout provides protection from nutrient stress.[69] We verified that the autophagic marker LC3B was upregulated basally in nCDase KO cells as compared to WT cells as was previously reported by Sundaram et. al. We wanted to also determine if pathways that regulate autophagy are altered basally in cells deficient in nCDase. To this end, we performed western blot analysis of protein kinase B (AKT) and glycogen synthase kinase 3 beta (GSK3ß) in MEF wild type and nCDase knockout cells. Data revealed upregulation of phosphorylated AKT (p-AKT) as well as increased phosphorylation of GSK3 $\beta$ (p-GSK3 $\beta$ ) (Fig 2). The increase in p-AKT along with $p$-GSK3 $\beta$ follows the reported regulation of GSK3 $\beta$ activity, as $p-A K T$ phosphorylates and inactivates GSK3 $\beta$. GSK3 $\beta$ inactivation or inhibition has been reported to upregulate pro-survival autophagy.[72, 73] It has also been reported that inhibition of GSK3 $\beta$ can protect from necrotic and apoptotic cell death by preventing mitochondrial permeability transition.[72] Although further characterization is needed to suggest these pathways are essential for nCDase knockout mediated protection from nutrient stress, literature suggests it could play a role. These processes would also likely be protective in the high-dose cisplatin model, as previous work has shown that autophagy plays a protective role.[47] 


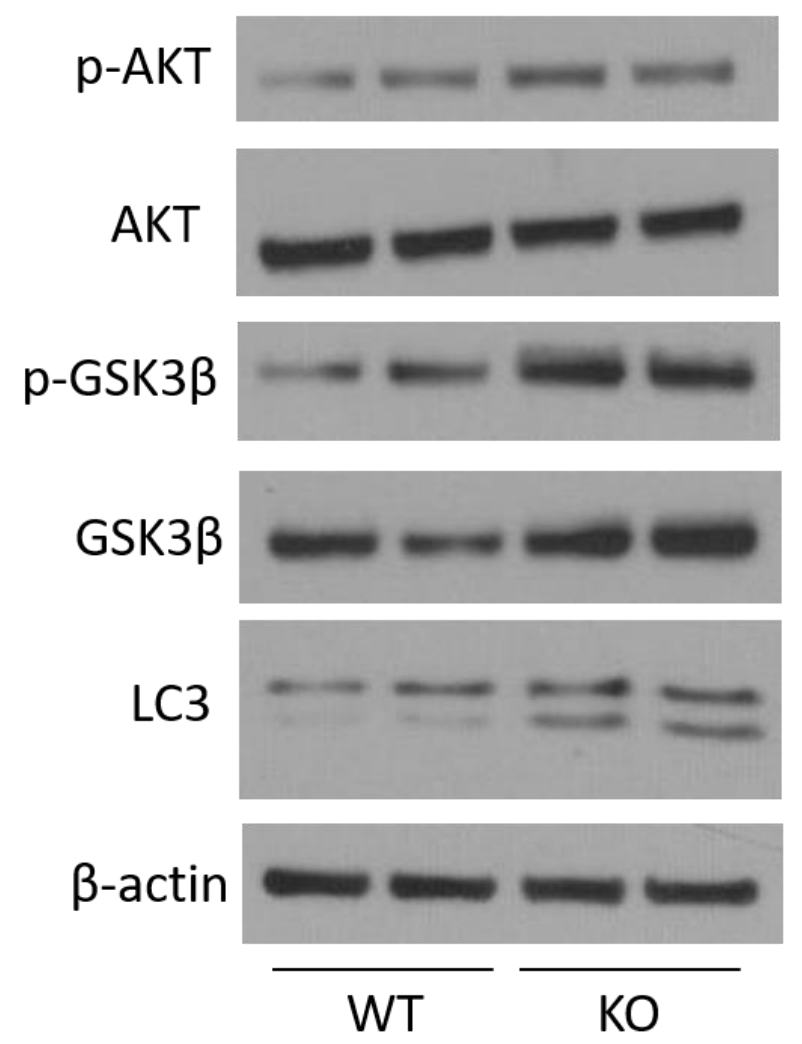

Figure 2. Upregulation of $p$-AKT and inhibition of GSK3 $\beta$ are induced by nCDase knockout.

Wild type and nCDase knockout MEFs were cultured in DMEM containing 10\% FBS. Cells were pelleted and lysed with cell extraction buffer containing protease and phosphatase inhibitors. $20 \mu \mathrm{g}$ of protein were then taken for western blot analysis. WT indicates wild type MEFs, KO indicates nCDase knockout MEFs. 


\section{Pharmacological inhibition of nCDase induces signaling changes as seen}

in genetically modified MEFs. In order to test whether signaling changes observed in nCDase knockout MEFs were due to long term compensation or acute inhibition of nCDase, we used two pharmacological inhibitors of nCDase in wild type MEF cells in vitro. C6-urea ceramide (C6) is a widely used inhibitor of nCDase but is known to have high levels of toxicity due to off target effects. We also used a newly developed nCDase inhibitor from Ono Pharmaceutical Company (ONO). We wanted to inhibit nCDase at a concentration that was not lethal to cells yet still provided adequate enzymatic inhibition. Viability of MEF wild type cells was assessed under treatment with a wide range of concentrations of both ONO and C6 drug over a period of 72 hours. The ONO drug was shown to be less toxic then $\mathrm{C} 6$ (Fig $3 a$ and $3 b$ ). At $50 \mu \mathrm{M}$, ONO treated wild type MEFs still displayed around 50\% viability. At $50 \mu \mathrm{M}, \mathrm{C} 6$ killed all wild type MEFs by 24 hours. We also observed that ONO showed some selectivity in killing wild type MEFs more effectively than nCDase knockouts (Fig 3a and 3b). This indicates ONO may be acting in a more targeted manner. We also tested the activity of the drugs in vitro, using a TLC enzymatic activity assay with recombinant nCDase (Fig 3c, Fig 3d). From these experiments, we identified a smaller range of concentrations where nCDase inhibition was observed. Western blot analysis of MEFs treated at these concentrations of inhibitor revealed that pharmacologic inhibition of nCDase resulted in upregulation of $p-A K T, p-G S K 3 \beta$, and LC3 as previously seen with genetic knockout of nCDase (Fig 4). This 
suggests that these effects may be due to acute inhibition of nCDase activity and not long-term compensation. 

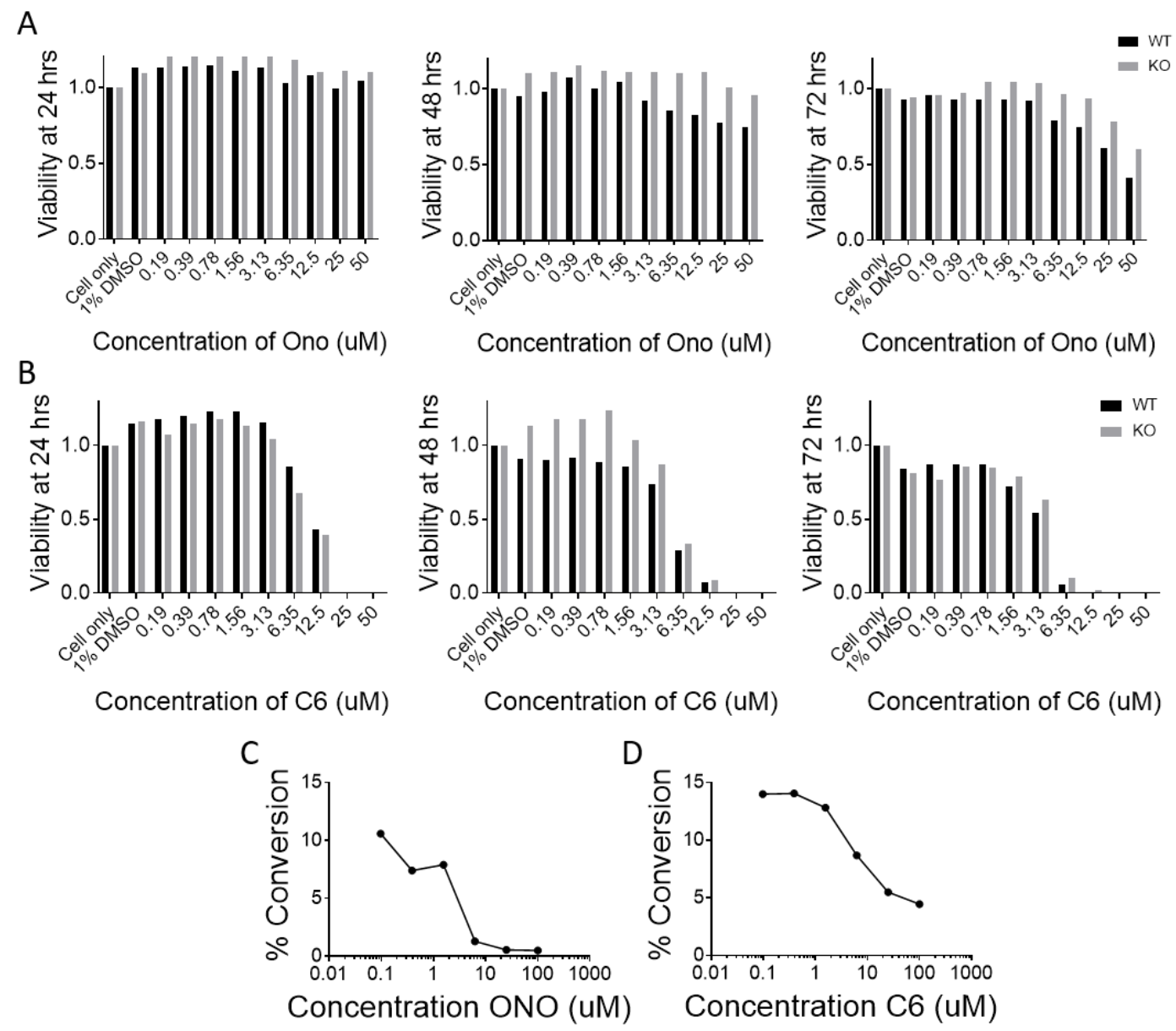

Figure 3. Viability and nCDase enzymatic activity of wild type MEFs treated with pharmacologic inhibitors of $\mathbf{n C D a s e .}$

(A,B) Wild type MEFs were plated at 1,000 cells per well in a 96-well plate. Ono drug (3a) or C6 (3b) concentrations were added the next day. Viability was monitored via alamar blue assay over 72 hours. WT indicates wild type MEFs, KO indicates nCDase knockout MEFs (C,D) nCDase inhibition was assessed using an in vitro, TLC activity assay of wild type MEFs treated with inhibtiors. Inhibition of ceramide conversion to sphingosine was observed under both Ono (3c) and C6 (3d) treatment. 


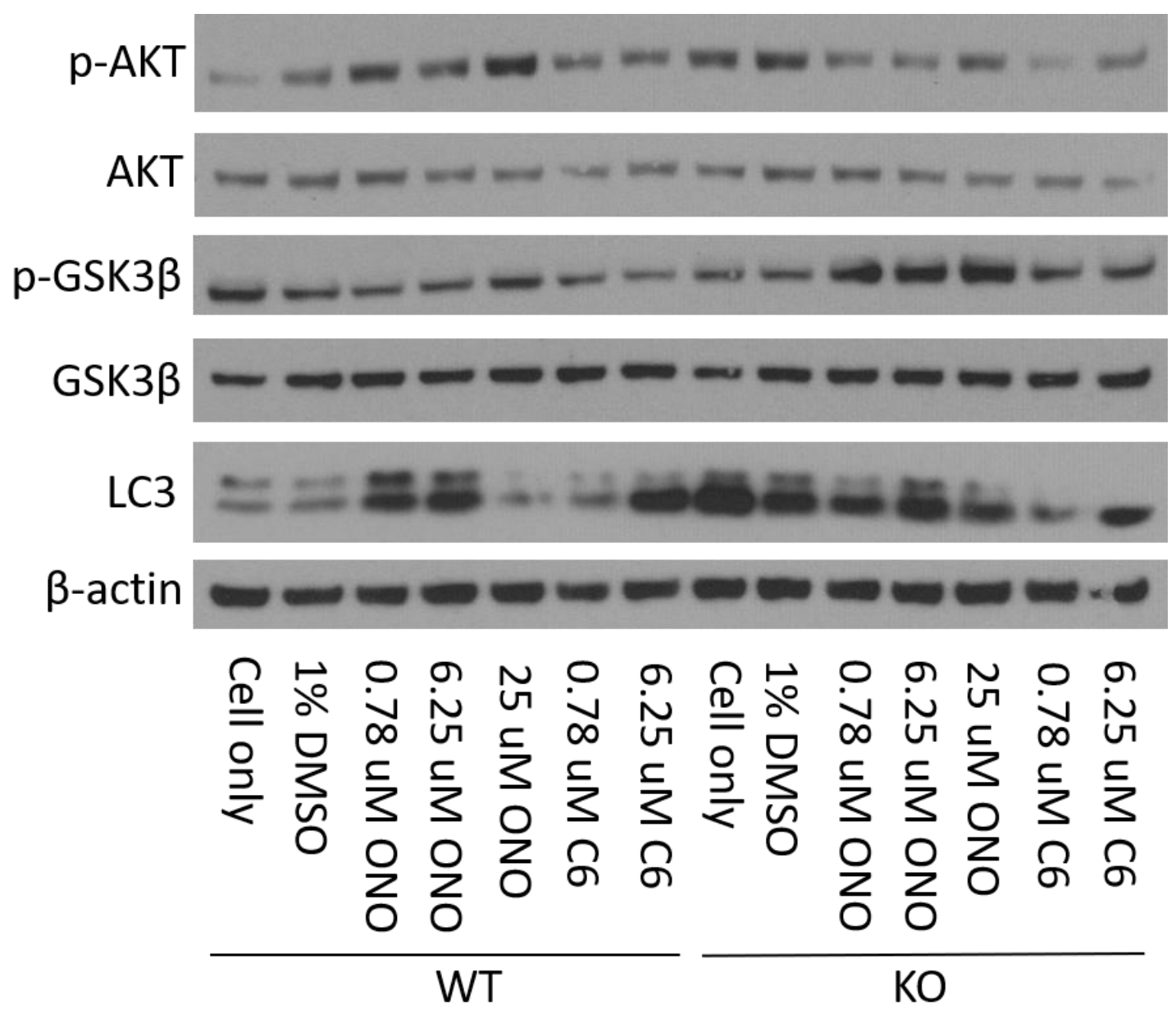

Figure 4. Acute inhibition of nCDase confers signaling changes as seen in nCDase knockout MEFs.

Wild type and nCDase knockout MEFs were cultured for 24 hours in the presence of either Ono or C6 drug. Cells were then harvested and lysed in cell extraction buffer containing protease and phosphatase inhibitors. $20 \mu \mathrm{g}$ of protein were then analyzed for each condition via western blot. WT indicates wild type MEFs, KO indicates nCDase knockout MEFs. 


\section{Loss of nCDase confers resistance to AKI following high-dose cisplatin}

treatment. Our in vitro results above and elsewhere[69] indicated that nCDase knockout protects MEFs from ER stress and nutrient deprivation via upregulation of autophagy. Upregulation of autophagy has been reported to be protective in acute models of CDDP-KI.[70] In order to test the effects of nCDase on the development of AKI following acute, high-dose cisplatin treatment, we utilized a colony of $\mathrm{C} 57 \mathrm{BL} / 6$ mice with a neo cassette inserted into the encoding gene of $\mathrm{nCDase}$. As a result, mice homozygous for this gene produce a nonfunctional $\mathrm{nCDase}(\mathrm{nCD}$ ase -/-). We found that genetic inhibition of $\mathrm{nCD}$ ase in vivo also leads to an increase in the LC3B marker of autophagy in the kidney cortex (Fig 5). Following treatment with high dose cisplatin, nCDase -/- mice displayed resistance to acute kidney injury development. Kidney function as measured by BUN was significantly lower in nCDase -/- mice 72 hours post cisplatin treatment (Fig 6a). Serum creatinine (SCr) and NGAL levels also appeared lower in nCDase -/- mice compared to wild type although these results were not significant (Fig 6b, Fig 6c). nCDase -/- mice had significantly lower levels of tubular necrosis, cast formation, inflammatory cells, and loss of brush border as determined by pathology. Tubular dilation and tubule degeneration was also lower in nCDase -/- mice compared to wild type although these results were not significant (Fig 7b-g). Inflammatory cytokine production following cisplatin treatment was also assessed using qRT-PCR of kidney tissue. TNFa, IL6, MCP1, and CXCL1 levels were lower in nCDase -/- mice but were not statistically different from wild type (Fig 8a-d). 


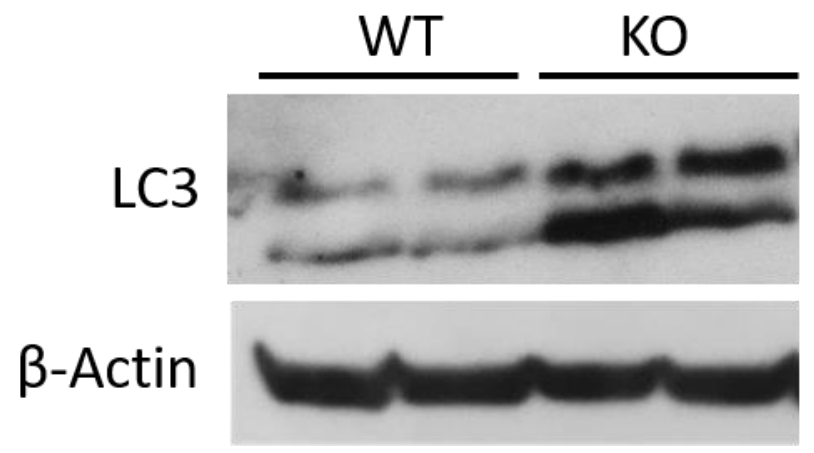

Figure 5. nCDase -/- mice display increased LC3 expression compared to wild type.

Renal cortex and medulla from vehicle treated wild type and nCDase -/- mice were harvested and prepped for western blot analysis. WT indicates wild type mice, KO indicates nCDase -/- mice. Experiment done by Dr. Tess Dupre. 

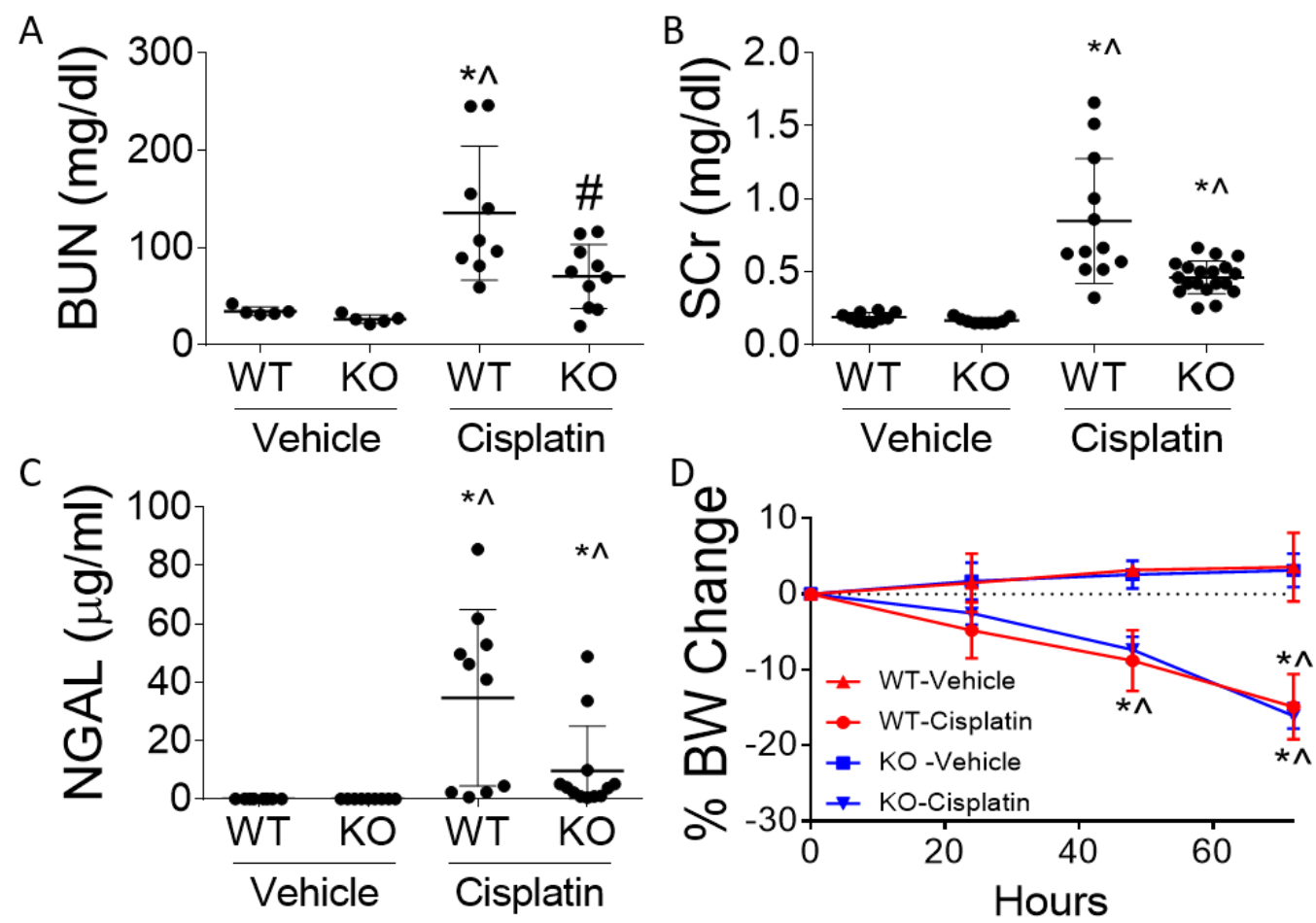

Figure 6. Loss of nCDase attenuates AKI following high-dose cisplatin treatment.

C57BL/6 wild type and nCDase -/- mice were injected (i.p.) with either $20 \mathrm{mg} / \mathrm{kg}$ cisplatin or normal saline. Mice were sacrificed 72 hours after cisplatin treatment and kidney injury markers were assessed. $(A, B) B U N$ and SCr were measured from serum of cisplatin and vehicle treated wild type and nCDase -/- mice. (C) NGAL levels were assessed from urine of cisplatin and vehicle treated wild type and nCDase -/- mice. (D) Mouse body weight was measured each day following cisplatin treatment. * signifies statistically different than vehicle treated WT mice. \# signifies statistically different than cisplatin treated WT mice. ${ }^{\wedge}$ signifies statistically different than vehicle treated KO mice. Experiment was performed by Dr. Tess Dupre, data were reanalyzed by Sophia Sears. 

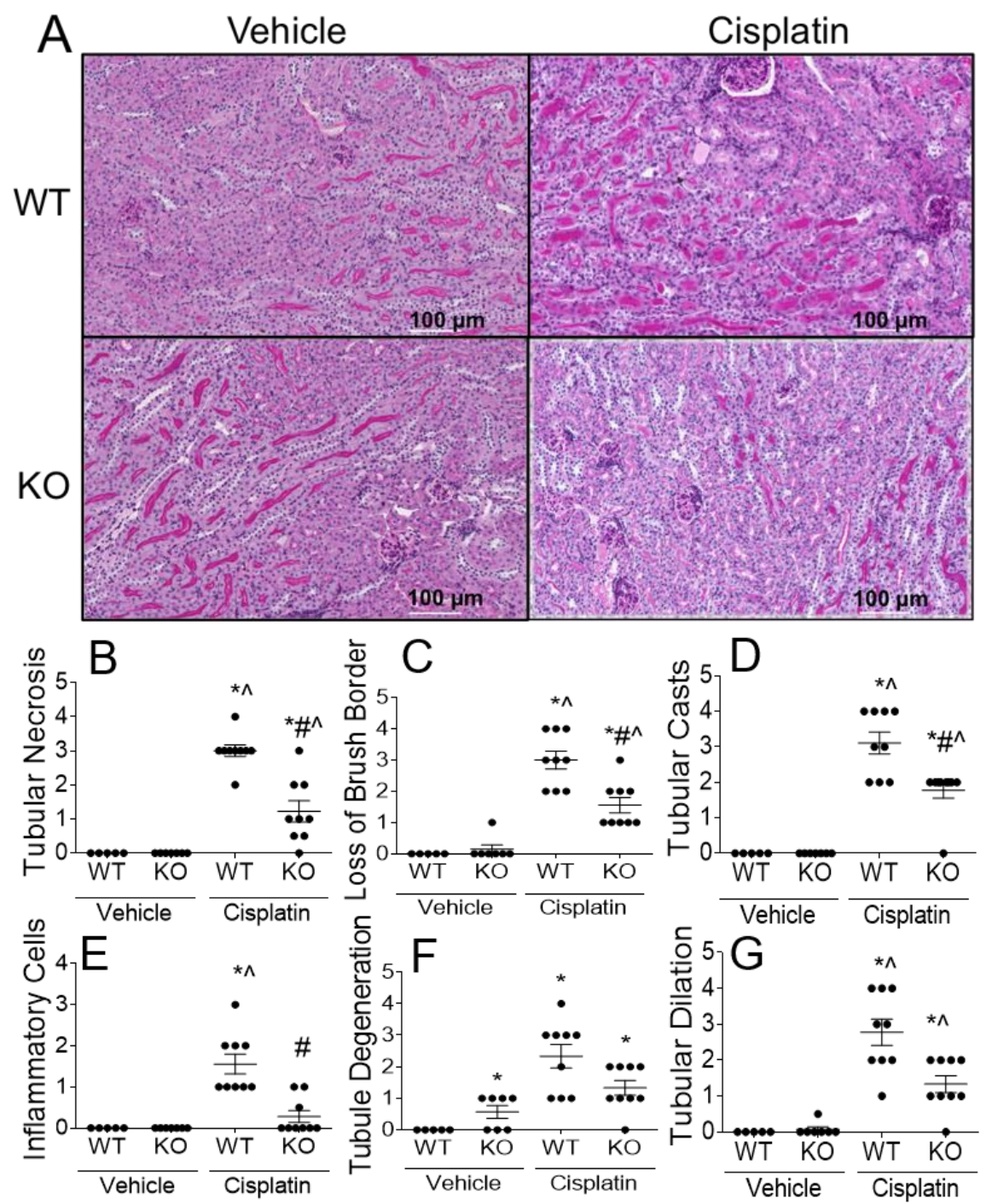

Figure 7. Loss of nCDase protects from high-dose cisplatin-induced deterioration in kidney pathology.

C57BL/6 wild type and nCDase -/- mice were injected (i.p.) with either $20 \mathrm{mg} / \mathrm{kg}$ cisplatin or normal saline. Mice were sacrificed 72 hours after cisplatin treatment. Renal histological changes were assessed on H\&E and PAS stained sections 5 $\mu \mathrm{m}$ thick. (A) Representative images of renal histology at 200X magnification. (B) Tubular necrosis, (C) loss of proximal tubule brush borders, (D) proximal tubule cast formation, $(E)$ inflammatory cells, $(F)$ tubule degeneration, and $(G)$ tubule dilation were assessed as markers of histological changes. For figures (B-G), scoring of the sections was performed in a blinded manner by renal pathologist Dr. Megyesi using a scale of 0-4 ( $0=$ not present, $1=$ mild, $2=$ moderate, $3=$ severe, and $4=$ very severe renal histological changes in the proximal tubules). WT indicates wild type mice, $\mathrm{KO}$ indicates $\mathrm{nCDase}{ }^{-/}$mice. ${ }^{*}$ signifies statistically different than vehicle treated WT mice. \# signifies statistically different than cisplatin treated WT mice. ${ }^{\wedge}$ signifies statistically different than vehicle treated KO mice. Experiment was performed by Dr. Tess Dupre, data were reanalyzed by Sophia Sears. 

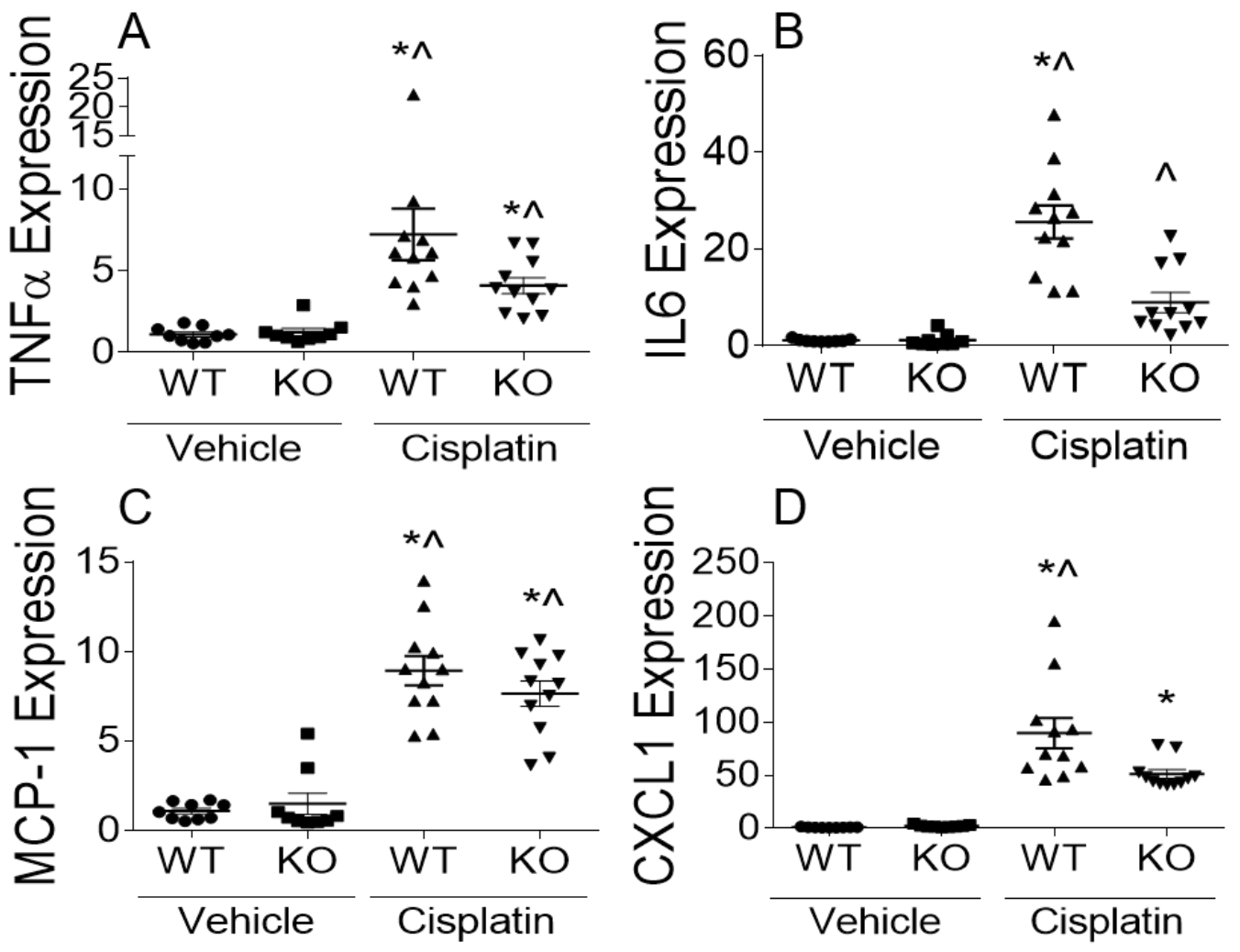

Figure 8. Loss of nCDase decreases mRNA expression of inflammatory cytokines in the kidney following high-dose cisplatin treatment.

C57BL/6 wild type and nCDase -/- mice were injected (i.p.) with either $20 \mathrm{mg} / \mathrm{kg}$ cisplatin or normal saline. Mice were sacrificed 72 hours after cisplatin treatment. Relative expression of (A) TNFa, (B) IL6, (C) MCP-1, and (D) CXCL1 mRNA were measured via qRT-PCR. WT indicates wild type mice, $\mathrm{KO}$ indicates $\mathrm{nCDase}{ }^{-/}$mice. ${ }^{*}$ signifies statistically different than vehicle treated WT mice. \# signifies statistically different than cisplatin treated WT mice. ^ signifies statistically different than vehicle treated KO mice. Experiment done by Dr. Tess Dupre, data were reanalyzed by Sophia Sears. 
nCDase -/- mice have reduced apoptosis following high-dose cisplatin treatment. It is well established that the acute, high-dose model of cisplatininduced kidney injury causes a high level of apoptosis. This high level of cell death mediates the pathological changes that manifest as acute kidney injury. We hypothesized that nCDase -/- may mediate protection from high-dose cisplatin by preventing cell death, as previously seen in our in vitro models of cell stress. In order to assess the level of apoptosis occurring in vivo, we performed a western blot of cleaved caspase 3. Following cisplatin treatment nCDase -/- mice had no detectable expression of cleaved caspase 3 (CC3) while wild type mice showed increased levels of CC3 as compared to vehicle treated mice (Fig 9a). We also performed TUNEL staining on wild type and nCDase -/- mice following cisplatin treatment. We found nCDase -/- mice treated with cisplatin had significantly less TUNEL-positive apoptotic cells then wild type treated mice (Fig 9b). These data indicate that nCDase -/- blocks induction of apoptotic cell death typically triggered by high-dose cisplatin. 


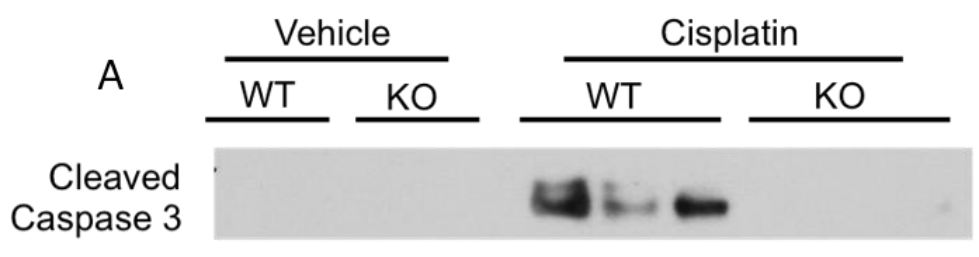

Actin
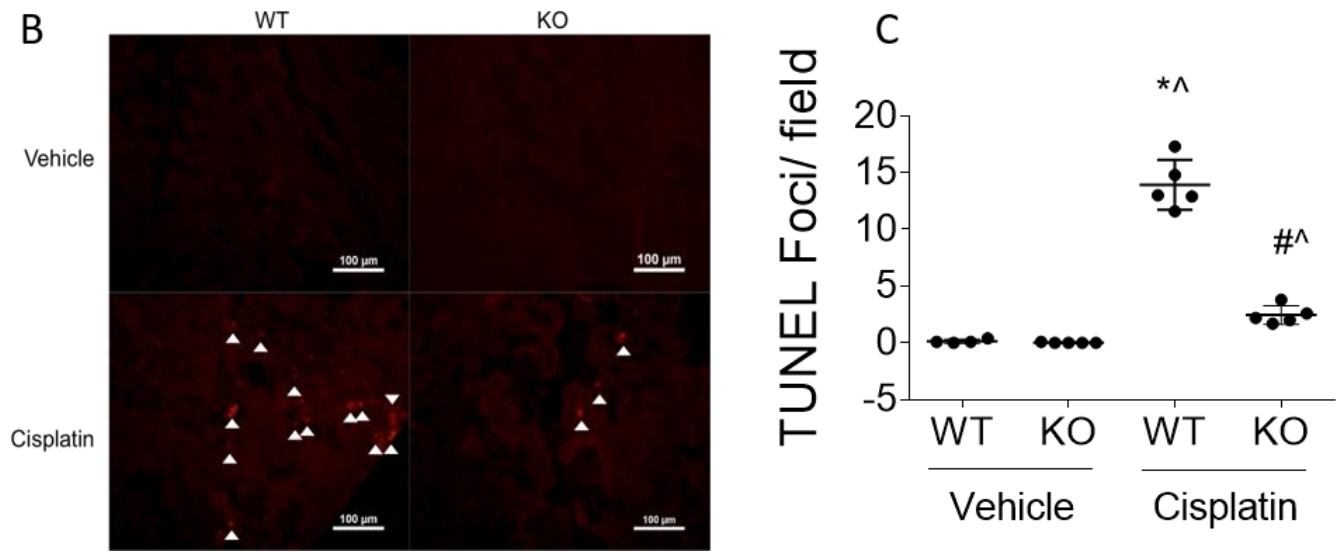

Figure 9. Loss of $\mathbf{n C D a s e}$ attenuates high-dose cisplatin-induced apoptosis.

C57BL/6 wild type and nCDase -/- mice were injected (i.p.) with either $20 \mathrm{mg} / \mathrm{kg}$ cisplatin or normal saline. Mice were sacrificed 72 hours after cisplatin treatment. (A) Western blot analysis was performed to assess relative protein levels of cleaved caspase 3 in the renal cortex of mice. (B-C) TUNEL assays were performed on paraffin embedded kidney sections as an index of apoptosis. (B) Representative photomicrographs of TUNEL assays (final magnification 200X). (C) TUNEL quantification. WT indicates wild type mice, KO indicates nCDase-- mice. ${ }^{*}$ signifies statistically different than vehicle treated WT mice. \# signifies statistically different than cisplatin treated WT mice. ${ }^{\wedge}$ signifies statistically different than vehicle treated KO mice. Experiment done by Dr. Tess Dupre. 
nCDase -/- mice have reduced levels of ER stress following high-dose cisplatin treatment. Cisplatin has been shown to induce ER stress as a mechanism of cell toxicity.[50] In order to assess how nCDase -/- would affect induction of ER stress we performed western blot analysis on wild type and nCDase -/- mice treated with cisplatin or vehicle. We found the nCDase -/- mice treated with cisplatin had reduced levels of ER stress markers p-JNK, CHOP, and p-elF2 $\alpha$ compared to wildtype mice treated with cisplatin (Fig 10). These data indicate that nCDase -/- blocks high-dose cisplatin-induced ER stress. 


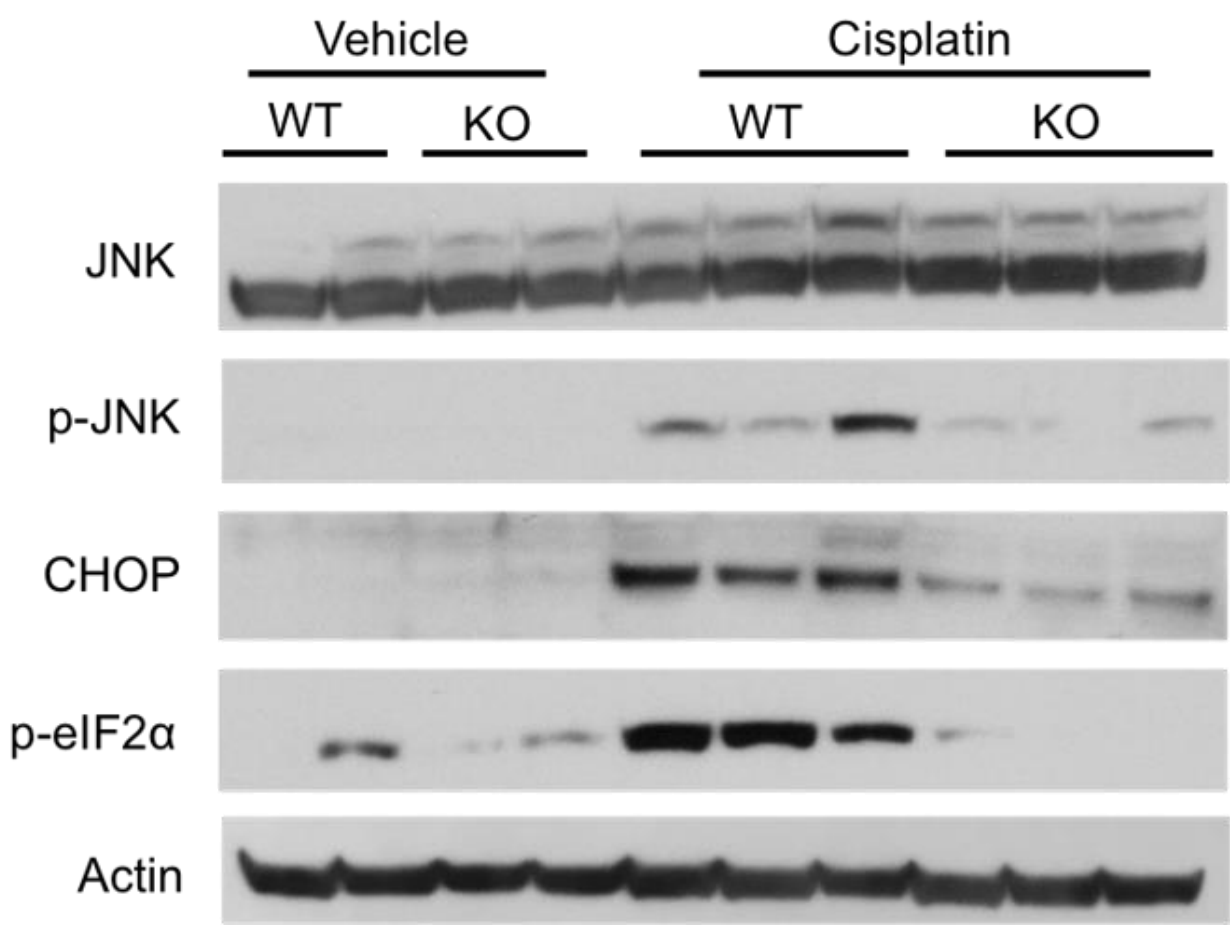

Figure 10. Loss of nCDase attenuates high-dose cisplatin-induced ER stress.

C57BL/6 wild type and nCDase -/- mice were injected (i.p.) with either $20 \mathrm{mg} / \mathrm{kg}$ cisplatin or normal saline. Mice were sacrificed 72 hours after cisplatin treatment. Western blot analysis was performed to assess relative protein levels of markers of ER stress in the renal cortex of mice. WT indicates wild type mice, KO indicates nCDase ${ }^{-/}$mice. Experiment done by Dr. Tess Dupre. 
Repeated low-dose cisplatin (RLDC) induces signaling pathways similar to nCDase -/- triggered pathways. As more information is being revealed about the interconnectedness of $\mathrm{AKI}$ and $\mathrm{CKD}$, it is becoming increasingly important to test prospective nephroprotective agents in models of both types of injury. With the development of our RLDC model, we had the capability to test nCDase as a target for cisplatin-induced renal fibrosis and CKD. As the pathology associated with our RLDC model is somewhat different than what is seen in the high-dose model, we were unsure if $\mathrm{nCDase}$-/- would mediate protection. However, upon analysis of wild type mice treated with RLDC we found signaling pathways were altered in a manner consistent with nCDase -/- mediated signaling changes in vitro. p-AKT, p-GSK3 $\beta$, and LC3 expression was upregulated in wild type mice following RLDC (Fig 11). Therefore, we hypothesized that nCDase -/- may confer resistance to RLDC induced kidney injury. 


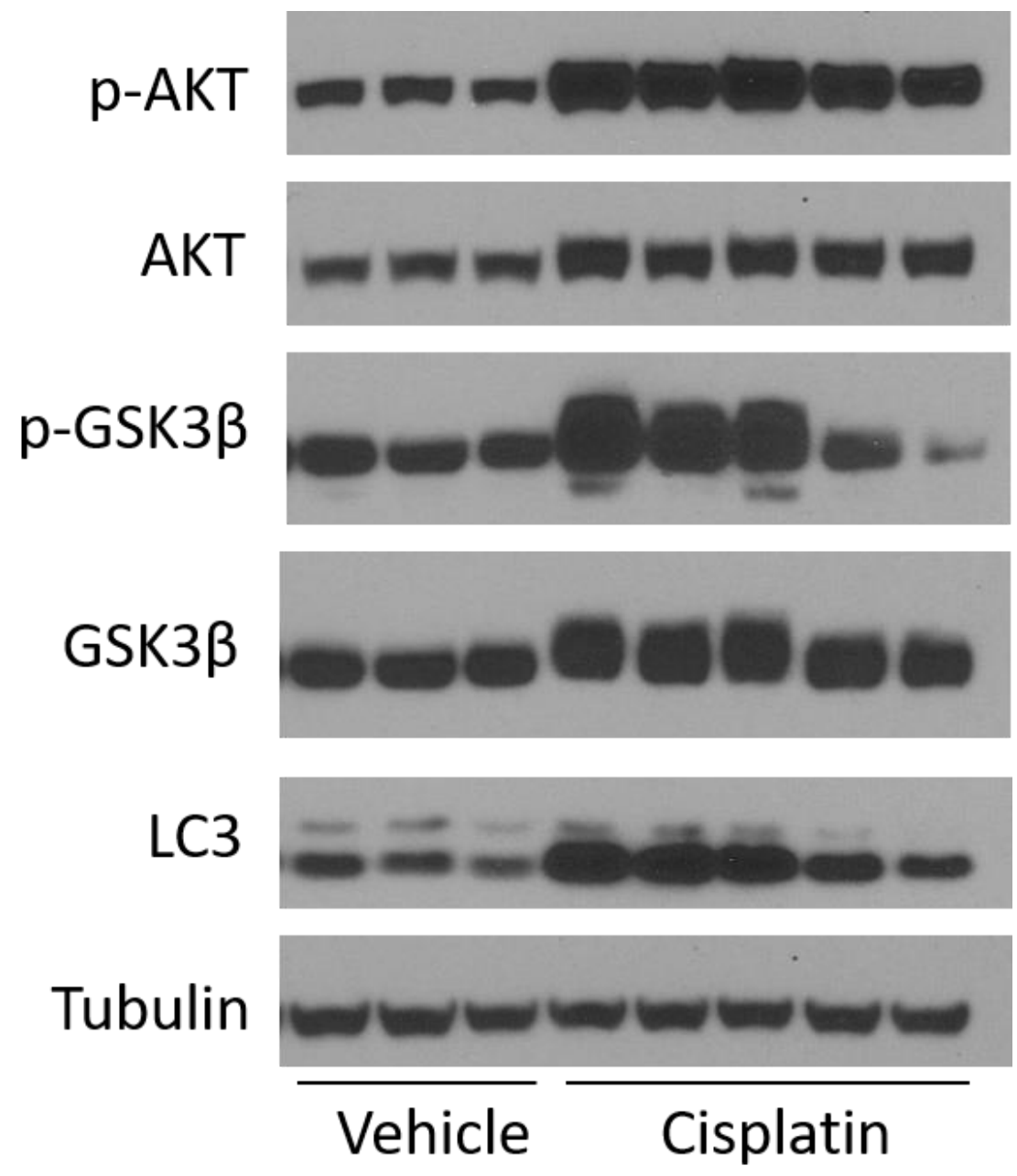

Figure 11. RLDC treatment of C57BL/6 mice induces changes in protein expression similar to those triggered by nCDase inhibition in vitro.

Wild type C57BL/6 mice were injected (i.p.) with either $9 \mathrm{mg} / \mathrm{kg}$ cisplatin or normal saline once a week for four weeks. Mice were sacrificed 72 hours following the final dose. Expression of the indicated proteins was evaluated from renal cortex using western blot. 


\section{nCDase inhibition does not protect from RLDC induced kidney injury.}

C57BL/6 wild type, nCDase -/-, and nCDase -/+ mice were injected (i.p.) with 9 $\mathrm{mg} / \mathrm{kg}$ cisplatin or saline once a week for four weeks. $500 \mu \mathrm{l}$ saline was injected (subQ) 1 day before dose 3, two days after dose 3, and 1 day before dose 4 . Mice were sacrificed 72 hours following the last dose. Kidney function was evaluated by BUN, NGAL, and Kim-1 levels. Following RLDC there appeared to be no difference in BUN, NGAL, or Kim-1 levels between all cisplatin treated groups. All cisplatin treated groups had significantly elevated BUN, NGAL, and Kim-1 levels compared to vehicle treated controls (Fig 12a-c). Throughout RLDC, mouse body weights were monitored. Saline was administered to ensure weight loss did not fall below $25 \%$. All cisplatin treated mice appeared to have equal amounts of weight loss throughout RLDC regardless of their genotype (Fig 12d). Upon pathology assessment, nCDase -/-, nCDase -/+, and wild type mice appeared to have similar levels of damage in the measures assessed (Fig 13). Cisplatin treatment unexpectedly caused significant increases in tubular necrosis and tubular regeneration not seen in FVB mice under RLDC treatment.[57] Inflammatory cytokine expression following RLDC also indicated nCDase inhibition did not provide a protective effect. TNFa, IL6, MCP-1, and CXCL1 mRNA expression were similar between all cisplatin treated groups (Fig 14a-d). We also evaluated iNOS and Arg-1 mRNA expression to assess the level of M1 and M2 macrophages present. Genotype did not appear to influence iNOS or Arg-1 levels. Cisplatin treatment caused a significant decrease in iNOS expression and increase in Arg-1 expression as previously characterized (Fig 
14e-f). These data indicate that $n C D a s e-/-$ does not prevent renal function loss or injury development following RLDC. 
A

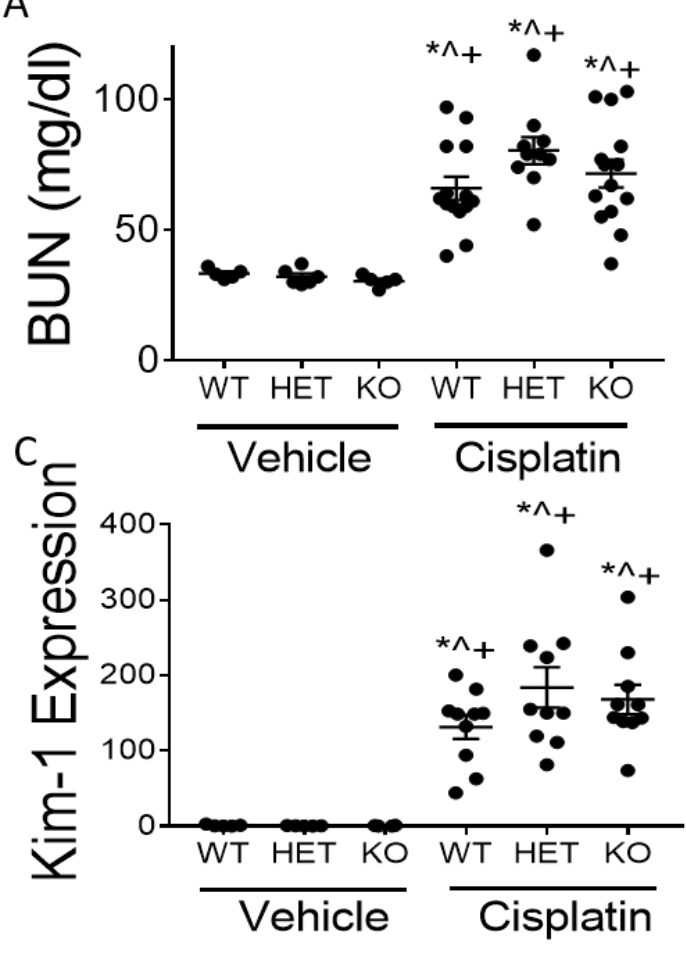

$\mathrm{B}$

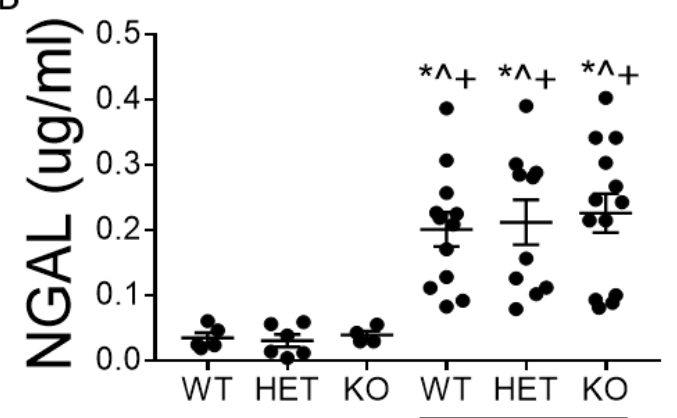

D Vehicle $\frac{\text { Cisplatin }}{\text { D }}$

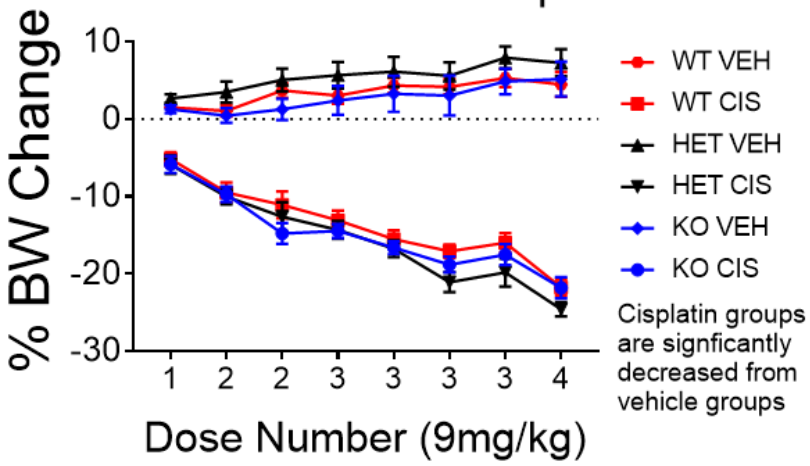

Figure 12. nCDase -/- does not prevent RLDC induced kidney injury.

C57BL/6 wild type, nCDase -/-, and nCDase -/+ mice were injected (i.p.) with either $9 \mathrm{mg} / \mathrm{kg}$ cisplatin or normal saline once a week for four weeks. $500 \mu$ l saline was injected (subQ) 1 day before dose 3, 2 days after dose 3 , and 1 day before dose 4 . Mice were sacrificed 72 hours following the final dose. (A) BUN levels were determined from serum. (B) NGAL levels were measured from the urine. (C) Kim-1 mRNA expression was measured via qRTPCR analysis of renal cortex. (D) Percent body weight change was tracked throughout RLDC as a measure of overt toxicity. WT indicates wild type mice, HET indicates nCDase /+ mice, KO indicates nCDase -/- mice. * signifies statistically different than vehicle treated WT mice. + signifies statistically different than vehicle treated HET mice. ^ ${ }^{\wedge}$ signifies statistically different than vehicle treated $\mathrm{KO}$ mice. 

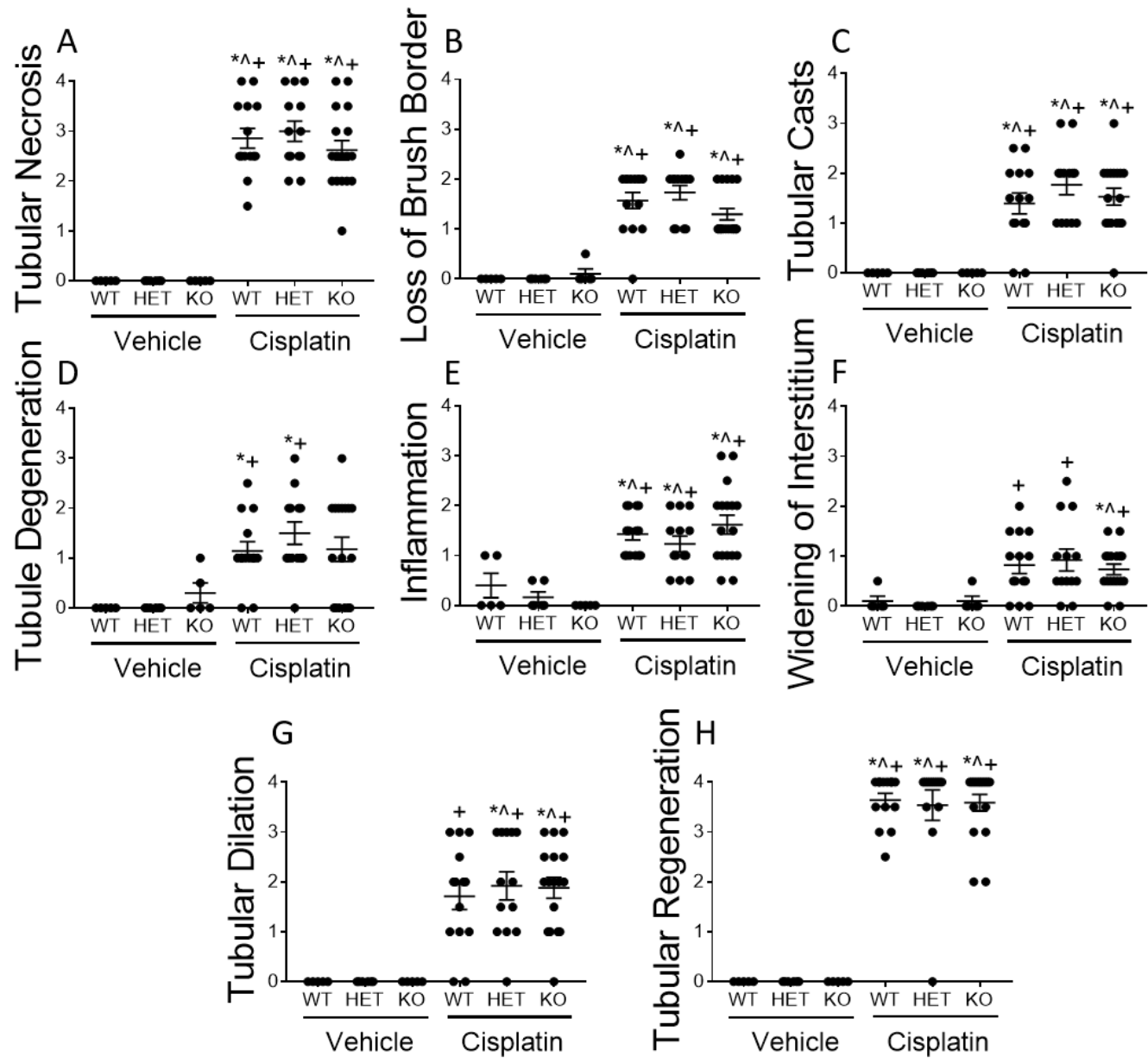

Figure 13. Loss of nCDase does not protect from RLDC induced renal pathology. C57BL/6 wild type, $\mathrm{nCDase}-/-$, and $\mathrm{nCDase}-/+$ mice were injected (i.p.) with either 9 $\mathrm{mg} / \mathrm{kg}$ cisplatin or normal saline once a week for four weeks. $500 \mu \mathrm{l}$ saline was injected (subQ) 1 day before dose 3, 2 days after dose 3, and 1 day before dose 4 . Mice were sacrificed 72 hours following the final dose. Renal histological changes were assessed on H\&E and PAS stained sections $5 \mu \mathrm{m}$ thick. (A) Tubular necrosis, (B) loss of proximal tubule brush borders, (C) proximal tubule cast formation, (D) tubule degeneration, $(E)$ inflammatory cells, $(F)$ widening of interstitium, $(G)$ tubule dilation, and $(\mathrm{H})$ tubular regeneration were assessed as markers of histological changes. Scoring of the sections was performed in a blinded manner by renal pathologist $\mathrm{Dr}$. Megyesi using a scale of 0-4 ( $0=$ not present, $1=$ mild, $2=$ moderate, $3=$ severe, and $4=v e r y$ severe renal histological changes in the proximal tubules). WT indicates wild type mice, HET indicates nCDase -/+ mice, KO indicates nCDase -/- mice. * signifies statistically different than vehicle treated WT mice. + signifies statistically different than vehicle treated HET mice. ^ signifies statistically different than vehicle treated KO mice. 
A

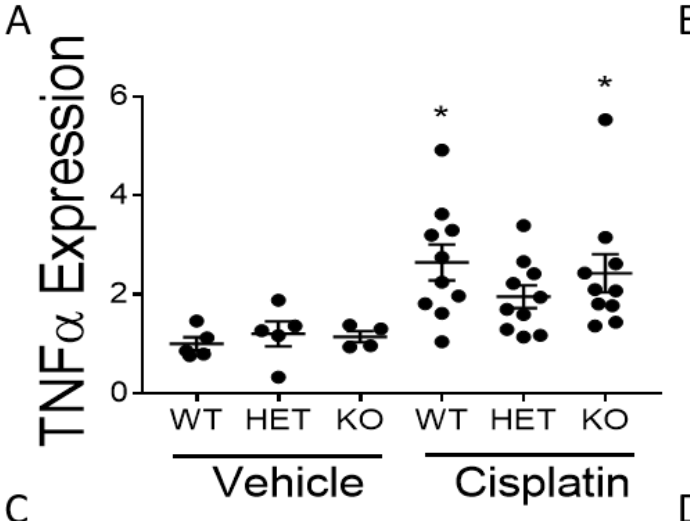

B
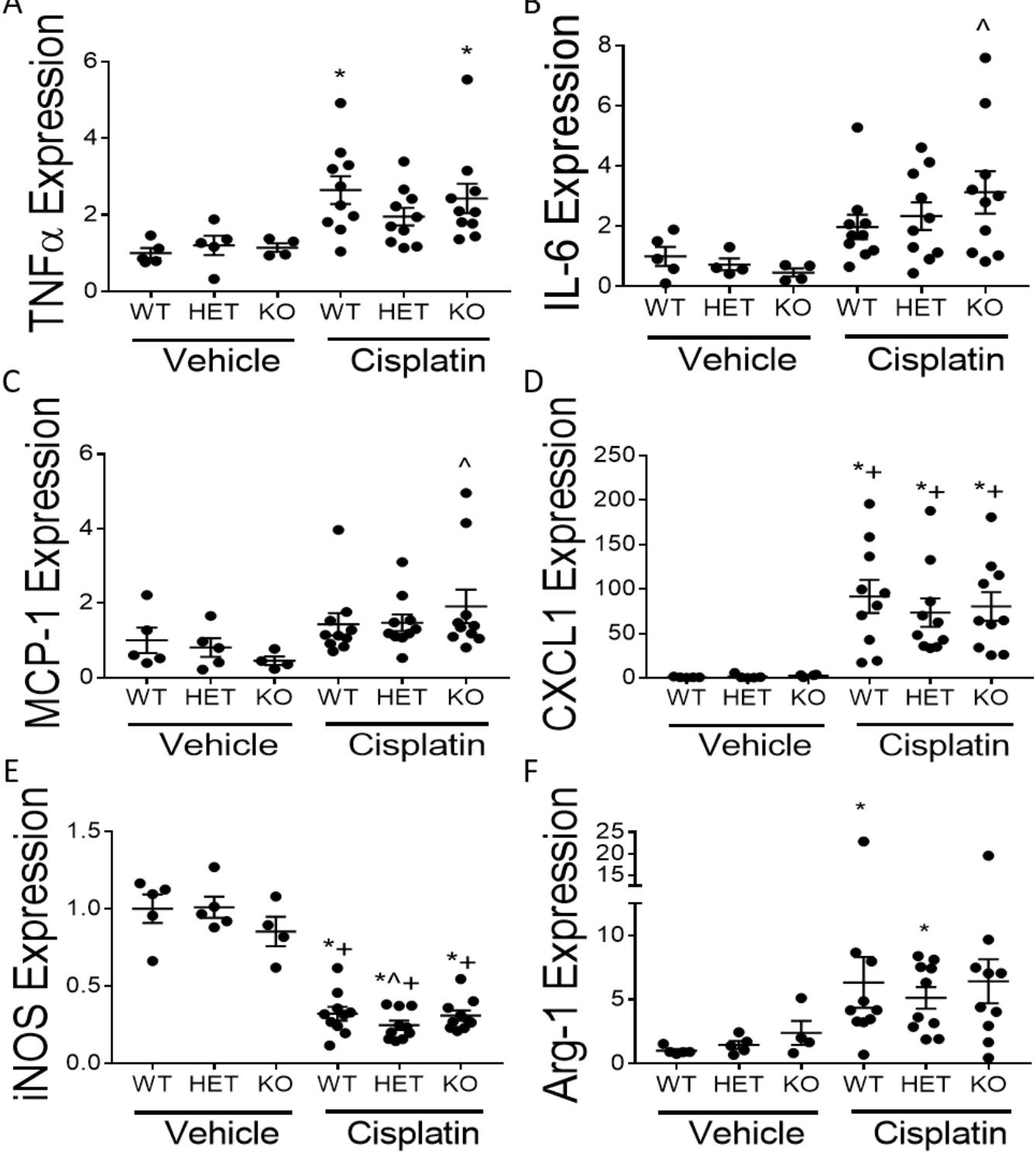

$\mathrm{F}$

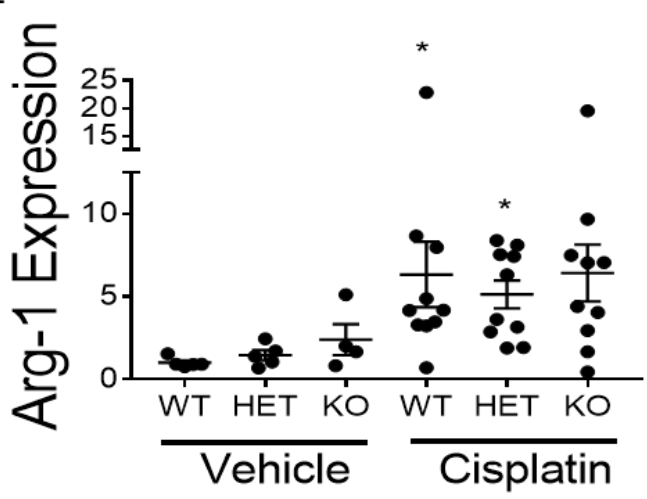

Figure 14. nCDase inhibition does not alter inflammatory cytokine production or macrophage involvement in RLDC.

C57BL/6 wild type, $\mathrm{nCDase}-/-$, and $\mathrm{nCDase}-/+$ mice were injected (i.p.) with either 9 $\mathrm{mg} / \mathrm{kg}$ cisplatin or normal saline once a week for four weeks. $500 \mu \mathrm{l}$ saline was injected (subQ) 1 day before dose 3, 2 days after dose 3, and 1 day before dose 4 . Mice were sacrificed 72 hours following the final dose. Relative mRNA expression of (A) TNFa, (B) IL6, (C) MCP-1, (D) CXCL1, (E) iNOS, and (F) Arg-1 were measured via real-time qRT-PCR. WT indicates wild type mice, HET indicates nCDase -/+ mice, KO indicates $\mathrm{nCDase}{ }^{-/}$mice. ${ }^{*}$ signifies statistically different than vehicle treated WT mice. + signifies statistically different than vehicle treated HET mice. ^ signifies statistically different than vehicle treated $\mathrm{KO}$ mice. 


\section{nCDase inhibition does not protect from development of fibrosis following}

RLDC. Previously, it had been shown that RLDC leads to collagen deposition and development of interstitial fibrosis.[57] We evaluated the development of fibrosis in wild type and nCDase -/- mice following RLDC using several methods. We detected levels of fibronectin expressed in the renal cortex using western blot. Our results suggest fibronectin expression is induced in both wild type and nCDase -/- mice in a similar fashion (Fig 15a). Sirius red fast green staining was also done to evaluate collagen deposition. In this stain, all collagen is dyed red while non-collagenous proteins are dyed green. Following RLDC, we saw similar amounts of collagen deposition in both wild type and nCDase -/- treated mice (Fig 15b). Fibrosis was also assessed by the pathological measure of widening of the interstitium. There was no difference in this measure among nCDase -/-, nCDase $-/+$, and wild type mice treated with cisplatin (Fig 13f). Interestingly, pathological assessment revealed our C57BL/6 mice may develop lower levels of fibrosis than what we've observed in FVB mice previously.[57] These data suggest nCDase does not play a role in development of fibrosis following RLDC. 
A

Fibronectin
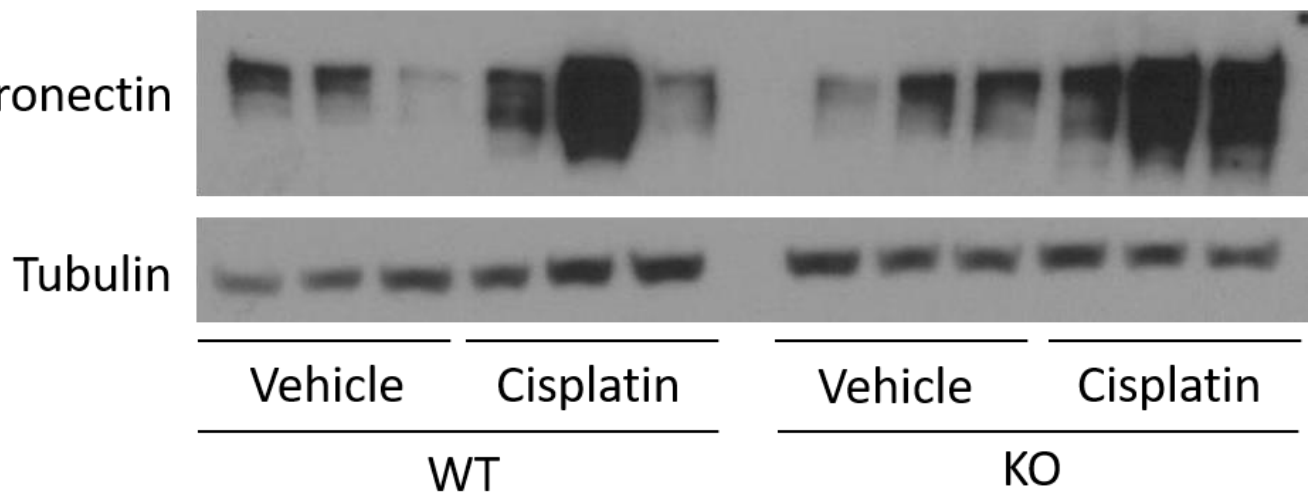

B

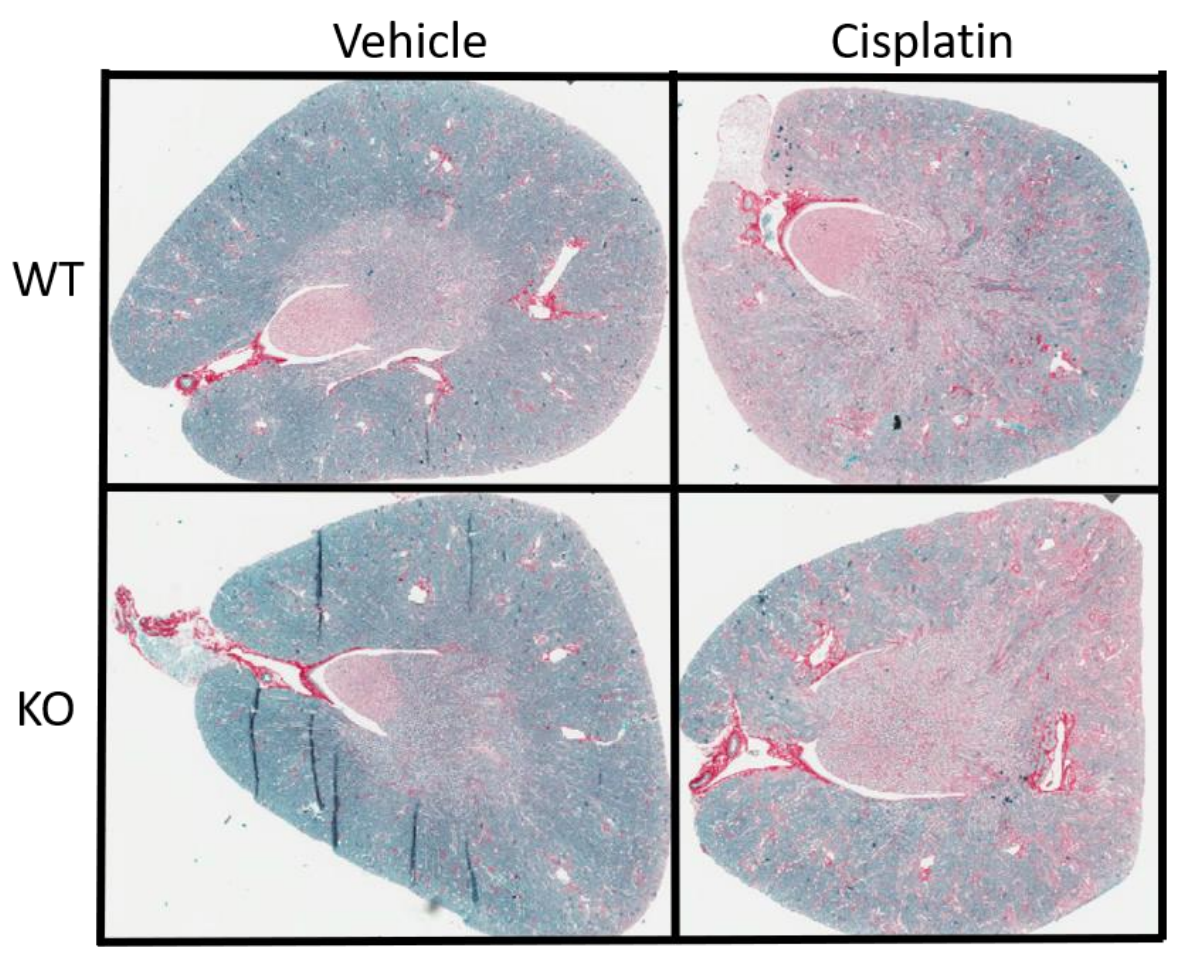

Figure 15. nCDase inhibition does not alter fibronectin expression or collagen deposition following RLDC.

C57BL/6 wild type and nCDase -/- mice were injected (i.p.) with either $9 \mathrm{mg} / \mathrm{kg}$ cisplatin or normal saline once a week for four weeks. $500 \mu \mathrm{l}$ saline was injected (subQ) 1 day before dose 3, 2 days after dose 3, and 1 day before dose 4 . Mice were sacrificed 72 hours following the final dose. (A) Protein expression of fibronectin in the renal cortex was evaluated via western blot. (B) Sirius red fast green staining was performed on nCDase -/- and wild type mice. WT indicates wild type mice, KO indicates $\mathrm{nCDase}{ }^{-/-}$mice. 
nCDase inhibition no longer attenuates cisplatin-induced ER stress. Having previously observed that nCDase -/- mice were resistant to ER stress in the highdose cisplatin model, we wanted to evaluate if this protection persisted in the RLDC model. We evaluated protein expression of ER stress markers CHOP, JNK, and NF-KB. Our data indicate that ER stress is induced in the RLDC model and nCDase inhibition does not block this induction (Fig 16). This suggests that RLDC employs different mechanisms of activating ER stress than what has previously been observed in the high-dose cisplatin model. 


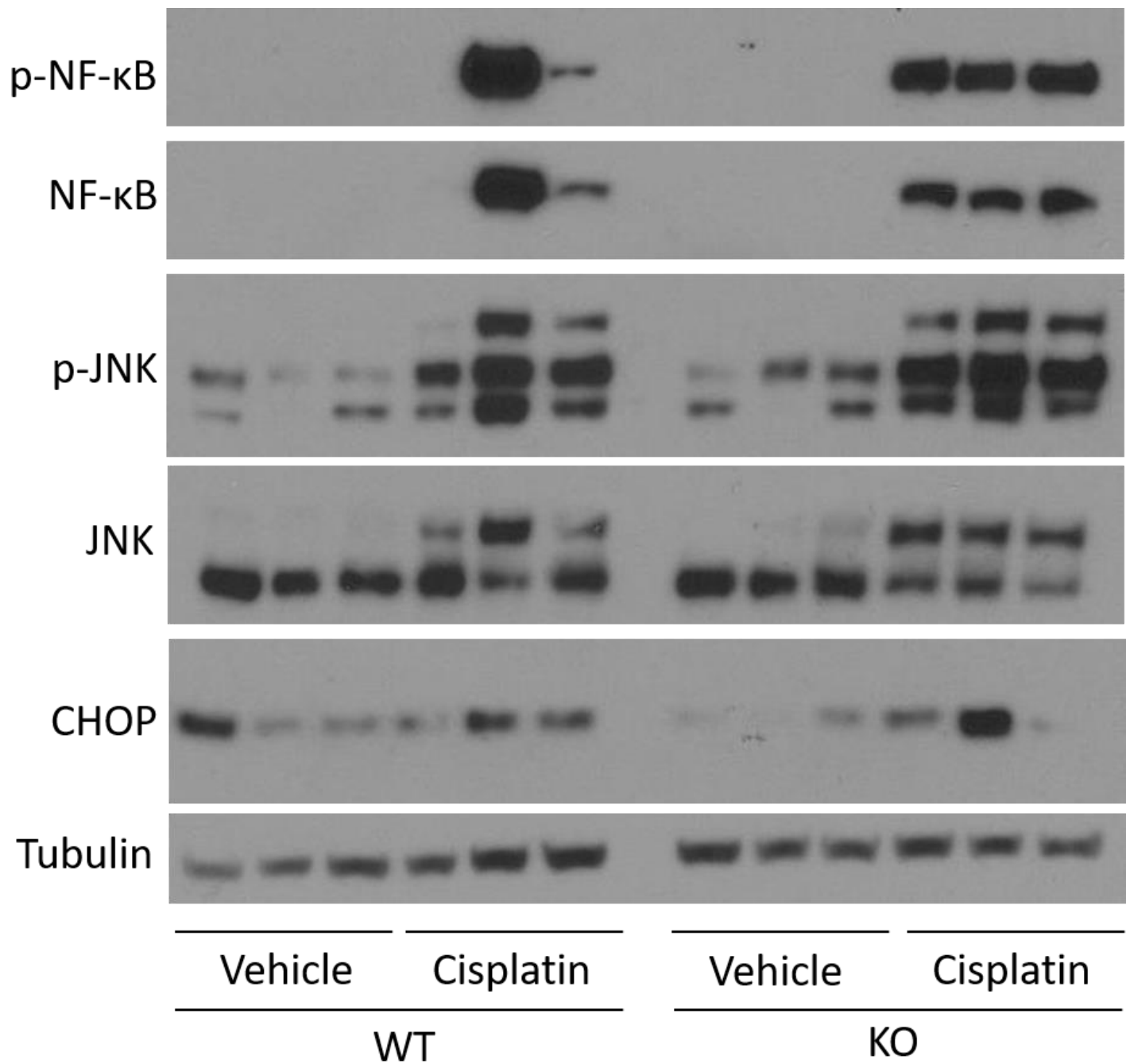

Figure 16. $\mathrm{nCDase}$-/- mice are not protected from ER stress in RLDC.

C57BL/6 wild type and nCDase -/- mice were injected (i.p.) with either $9 \mathrm{mg} / \mathrm{kg}$ cisplatin or normal saline once a week for four weeks. $500 \mu$ saline was injected (subQ) 1 day before dose 3, 2 days after dose 3 , and 1 day before dose 4 . Mice were sacrificed 72 hours following the final dose. (A) Protein expression of the indicated ER stress markers from the renal cortex was evaluated via western blot. WT indicates wild type mice, $\mathrm{KO}$ indicates $\mathrm{nCDase} \mathrm{e}^{-/}$mice. 


\section{DISCUSSION}

This study evaluates the role of neutral ceramidase ( $\mathrm{nCDase}$ ) in an in vitro system as well as in vivo in two different models of CDDP-KI. In vitro, nCDase knockout in mouse embryonic fibroblasts (MEFs) promoted increased autophagy and resistance to nutrient deprivation induced cell death. Our results in vivo indicate that nCDase knockout offers protection from high-dose cisplatin induced acute kidney injury (AKI) by preventing ER stress and apoptotic cell death. In the repeated low dose cisplatin (RLDC) model, nCDase knockout does not provide protection from kidney injury and development of fibrosis. nCDase knockout also does not block ER stress induction in the RLDC model.

These results indicate the importance of testing potential nephroprotective agents in both models of $\mathrm{AKI}$ and chronic kidney disease (CKD). It has been speculated that the RLDC model of injury employs the same mechanisms as the standard high-dose model. Here, we demonstrate that that is not likely the case. If the same mechanism of nephrotoxicity was produced in both models, nCDase knockout would have been protective in RLDC. The lack of protection in this model can give us clues into what mechanisms may be unique to RLDC induced nephrotoxicity.

In the high-dose model, AKI development is dependent on cell death. We have established $\mathrm{nCDase}$ knockout blocks cell death in both in vitro models of 
stress and in vivo in the high-dose model of cisplatin injury. Therefore, nCDase knockout provides protection from high-dose cisplatin. Previously, the RLDC model has not been shown to induce large levels of apoptosis or necrosis. [58] In this study, apoptosis was not measured but high levels of tubular necrosis were observed by pathology. We do not believe the driving pathology in RLDC induced fibrosis is cell death, but it could be a side effect of CKD development. In the high-dose model, cell death is the cause of AKI development. In the RLDC model, we believe cell death may be an effect of CKD development. Other studies have demonstrated how repeated sublethal injuries can lead to different renal outcomes than what is observed in acute models of kidney injury.

Grgic et. al. examined how multiple sublethal insults to tubule epithelial cells promotes interstitial fibrosis and other CKD pathologies. They utilized renal epithelial cells expressing a diphtheria toxin receptor. Administration of diphtheria toxin resulted in specific renal epithelial cell death. Using this model, they demonstrated that a large, acute insult to tubule cells led to an inflammatory response and tubule cell proliferation allowing for complete recovery. With repeated, lower levels of injury they observed that the kidney underwent maladaptive repair followed by development of fibrosis and glomerulosclerosis. They speculated that repeated injury caused tubule cells to lose their replicative capacity and resulted in either senescence or a prolonged dedifferentiated state.[74] Cell cycle G2/M arrest has been identified as a contributor to fibrotic outcomes in renal models of injury. G2/M cell cycle arrest is also associated with 
high levels of JNK signaling and secretion of profibrotic cytokines such as TGF $\beta .[21]$

Our current and past studies indicate that RLDC may also be causing cell cycle arrest and senescence. We see increased p-JNK protein expression (Fig 16)[58] and TGF $\beta$ mRNA expression [58] with RLDC. We have also observed in this study (data not shown) and in the past [58] that RLDC causes increased mRNA expression of cyclin dependent kinase inhibitor 2a (CDKN2A), a known marker of cellular senescence. Therefore, we hypothesize that RLDC induced fibrosis is being driven by G2/M cell cycle arrest of tubule cells as they attempt to proliferate and repair renal damage.

More studies are needed to verify whether tubule cells in the RLDC model are undergoing cellular senescence. We also need to examine levels of cell death at earlier timepoints in RLDC. These studies will help determine when senescence occurs and what cells are responsible. It could be that initially injured tubule cells remain and senesce, or there could be an initial cell death response to injury followed by uninjured tubule cell dedifferentiation and replication that is impaired upon repeated insults.

Another possibility for the differences in nCDase knockout outcomes in high-dose cisplatin and RLDC could be due to varied roles of autophagy in acute and chronic kidney injury. In the acute model of cisplatin injury, autophagy has been shown to be protective. Takahashi et. al. demonstrated that high-dose cisplatin treatment led to upregulation of autophagy in the kidney. They then treated mice with proximal tubule specific autophagy deficiency and found that 
they were more sensitive to injury. They also found that autophagy deficient mice had increased DNA damage, p53 activation, protein aggregation, and apoptosis following cisplatin treatment.[70] In this study, it is clear that autophagy protects against cellular damage induced by high dose cisplatin. Therefore, nCDase knockout would provide protection via upregulation of autophagy.

In chronic models of kidney injury, the role of autophagy is less well defined. Nam et. al. made use of mice with distal tubule specific conditional knockout of autophagy related 7 (Atg7). These mice had deficient autophagy in the renal distal tubule cells compared to controls. Following unilateral ureteral obstruction, these mice had increased levels of renal fibrosis. Distal tubule autophagy deficiency was also associated with increased TGF $\beta$ expression.[75] In contrast to this study, Livingston et. al. used mice with a proximal tubule specific conditional knockout of Atg7. They found that mice deficient in proximal tubule autophagy had decreased levels of renal fibrosis following unilateral ureteral obstruction. They also saw decreased levels of renal fibrosis in mice treated with pharmalogical inhibitors of autophagy, chloroquine and 3methyladenine, prior to injury. Interestingly, this group saw no effect on TGF $\beta$ expression in autophagy impaired groups following injury.[76] These studies and others indicate that the role of autophagy in renal fibrosis may be context and cell-type dependent.[52]

There are several experiments we could do in the future to determine the role of autophagy in our RLDC model. First, we must examine how RLDC alters autophagy. We could treat a GFP-LC3 mouse with RLDC and observe 
autophagic flux at different time points. Once we determine how RLDC influences autophagy, we could make use of both pharmalogical inhibitors and conditional knockout mice to inhibit autophagy at different points of RLDC. Following inhibition we could examine outcomes of renal fibrosis and myofibroblast production to determine what role autophagy may be playing in disease progression.

There is also a need to evaluate ER stress induction in both the high-dose and RLDC models. ER stress describes a disruption in ER homeostasis involving accumulation of misfolded proteins within the ER. This results in activation of the unfolded protein response (UPR) or cell death. The UPR is activated in an attempt to restore physiological function to the ER. However, long term activation of UPR can lead to permanent functional changes.[77]

In this study, we demonstrated that nCDase knockout was sufficient to block ER stress induction in the high-dose model. In the RLDC model, nCDase knockout no longer blocked this induction. This suggests that there are different activators of ER stress in these models. Induction of ER stress in the RLDC model also appears to be occurring independently of cell death. This suggests that there is an adaptive response to ER stress in the RLDC model.

ER stress involves several complex signaling cascades. There are three main proteins thought of as activators of ER stress: pancreatic endoplasmic reticulum kinase (PERK), inositol-requiring enzyme 1 $\alpha$ (IRE1 $\alpha)$, and activating transcription factor 6 (ATF6). Each of these proteins trigger pathways involved in regulation of UPR and apoptotic responses.[77] We hypothesize that the extent 
of activation in each of these arms may differ in high-dose cisplatin model and the RLDC model. This may account for the differential ER stress activation in nCDase knockout mice. It may also account for the difference in ER stress outcomes.[78] Future studies aimed at understanding the different drivers of ER stress in the two models are needed.

Once activated, ER stress can lead to outcomes ranging from apoptosis to adaptation. Responses to ER stress depend on both the amount of cellular damage done as well as the length of time the insult persists. With acute insults, cells only need to withstand stress for a short period of time. Recovery depends on how quickly the cell can mount the UPR and clear accumulated unfolded proteins. Following clearance cellular function can return to normal. With chronic stress, the cell must undergo a functional change that is more permanent. This functional change and prolonged UPR allows cells to adapt to the chronic stress and escape cell death.[78]

We hypothesize that in RLDC, tubule cells undergo functional changes in order to adapt to chronic ER stress. This adaptive response likely involves transcriptional upregulation of genes involved in protein folding and processing as well as alteration of metabolic function.[78] Chronic ER stress has previously been linked to CKD progression.[79] One study demonstrated that inhibition of ER stress following renal ischemia-reperfusion prevented development of fibrosis.[80] Future studies are needed utilizing the RLDC model in which ER stress is inhibited to determine its role in fibrosis development. 
Literature also reports a close relationship between ER stress and autophagy. There is evidence to support ER stress induction and inhibition of autophagy.[51] The UPR has been shown to induce autophagy through IRE1a and PERK signaling. This induction is thought to act as a pro-survival response, helping clear damaged cellular debris and restore ER homeostasis. However, it has also been demonstrated that ER stress induced autophagy can mediate cell death through caspase 8 activation.[77] In the high-dose cisplatin model, it is possible that the upregulation of autophagy in nCDase knockout mice facilitates resistance to ER stress induction. In the RLDC it is less clear how ER stress and autophagy are being regulated. Future studies aimed at understanding the role of autophagy in the RLDC model and the relationship between autophagy and ER stress are needed.

This study reveals how nephroprotective agents can have differential success in the high-dose cisplatin model and the RLDC model due to the inherent biological differences in these two models in the induction of cell death/senescence, ER stress induction and response, and autophagy. Evaluating the success of different therapeutic strategies in these models may help shed light on the different nephrotoxic mechanisms employed by high-dose cisplatin and RLDC treatment.

It is important to take into account both acute and chronic renal injury when developing nephroprotective agents. Patients exhibit a wide range of response to cisplatin in the clinic. Some develop AKI after a single low dose while others may receive multiple doses without displaying signs of clinical AKI. 
Developing an understanding of the biological processes occurring in both the high-dose cisplatin and RLDC models will help us understand the pathological processes occurring in all categories of patients. This will lead to better treatment of $\mathrm{AKI}$ and prevention of CKD. 


\section{SUMMARY}

Cisplatin is a commonly used chemotherapeutic agent with dose limiting nephrotoxicity. $10-20 \%$ of cancer patients will receive cisplatin as part of their treatment regimen. $30 \%$ of these patients will develop acute kidney injury (AKI), or a rapid decline in renal function. Even patients that do not develop AKI by clinical standards are still at risk for long term declines in renal function. Development of kidney injury greatly increases risk of mortality and development of chronic kidney disease (CKD).

AKI is associated with high levels of tubule cell death, robust inflammation, and vascular damage. This type of injury has been experimentally modeled by giving mice a single, high dose of cisplatin. Mice do not survive longer then 3-4 days after treatment. CKD is associated with interstitial fibrosis, chronic inflammation, and vascular rarefaction. Recent studies have brought to light the interconnectedness of $\mathrm{AKI}$ and $\mathrm{CKD}$ through processes of maladaptive repair. In order to develop better nephroprotective agents we must use models that allow for study of this progression following cisplatin treatment. Our repeated low dose cisplatin (RLDC) model utilizes 4 weekly low doses of cisplatin that enable mice to survive up to 6 months after treatment. This allows for evaluation of long-term progression to CKD. 
We used both the high-dose cisplatin and RLDC models to assess the effects of neutral ceramidase (nCDase) on renal injury. We found that nCDase knockout mice were protected from AKI development in the high-dose cisplatin model. In contrast, nCDase knockout mice were not protected from development of renal fibrosis in the RLDC model. These experiments demonstrate that the high-dose cisplatin and RLDC models utilize different mechanisms of nephrotoxicity. While the high-dose cisplatin model has been well characterized, less is known about the RLDC model. Future studies will be aimed at further characterizing cell death, cell cycle arrest, autophagy, and ER stress in the RLDC model. 


\section{REFERENCES}

1. Levey, A.S. and M.T. James, Acute Kidney Injury. Ann Intern Med, 2017. 167(9): p. ITC66-ITC80.

2. Negi, S., et al., Acute kidney injury: Epidemiology, outcomes, complications, and therapeutic strategies. Semin Dial, 2018. 31(5): p. 519527.

3. Doyle, J.F. and L.G. Forni, Acute kidney injury: short-term and long-term effects. Crit Care, 2016. 20(1): p. 188.

4. Basile, D.P., M.D. Anderson, and T.A. Sutton, Pathophysiology of acute kidney injury. Compr Physiol, 2012. 2(2): p. 1303-53.

5. Lopes, J.A. and S. Jorge, The RIFLE and AKIN classifications for acute kidney injury: a critical and comprehensive review. Clin Kidney J, 2013. 6(1): p. 8-14.

6. Van Biesen, W., R. Vanholder, and N. Lameire, Defining acute renal failure: RIFLE and beyond. Clin J Am Soc Nephrol, 2006. 1(6): p. 1314-9.

7. Martensson, J. and R. Bellomo, The rise and fall of NGAL in acute kidney injury. Blood Purif, 2014. 37(4): p. 304-10.

8. Castillo-Rodriguez, E., et al., Kidney Injury Marker 1 and Neutrophil Gelatinase-Associated Lipocalin in Chronic Kidney Disease. Nephron, 2017. 136(4): p. 263-267.

9. Sabbisetti, V.S., et al., Blood kidney injury molecule-1 is a biomarker of acute and chronic kidney injury and predicts progression to ESRD in type I diabetes. J Am Soc Nephrol, 2014. 25(10): p. 2177-86.

10. Rizvi, M.S. and K.B. Kashani, Biomarkers for Early Detection of Acute Kidney Injury. The Journal of Applied Laboratory Medicine: An AACC Publication, 2017. 2(3): p. 386-399.

11. Rahman, M., F. Shad, and M.C. Smith, Acute kidney injury: a guide to diagnosis and management. Am Fam Physician, 2012. 86(7): p. 631-9.

12. Bonventre, J.V. and L. Yang, Cellular pathophysiology of ischemic acute kidney injury. J Clin Invest, 2011. 121(11): p. 4210-21.

13. Ferenbach, D.A. and J.V. Bonventre, Mechanisms of maladaptive repair after AKI leading to accelerated kidney ageing and CKD. Nat Rev Nephrol, 2015. 11(5): p. 264-76.

14. Chawla, L.S., et al., The severity of acute kidney injury predicts progression to chronic kidney disease. Kidney Int, 2011. 79(12): p. 13619.

15. Levey, A.S. and J. Coresh, Chronic kidney disease. Lancet, 2012. 379(9811): p. 165-80.

16. Coresh, J., et al., Prevalence of chronic kidney disease in the United States. JAMA, 2007. 298(17): p. 2038-47. 
17. Coresh, J., et al., Action plan for determining and monitoring the prevalence of chronic kidney disease. Kidney Int Suppl (2011), 2017. 7(2): p. 63-70.

18. Thakar, C.V., et al., Acute kidney injury episodes and chronic kidney disease risk in diabetes mellitus. Clin J Am Soc Nephrol, 2011. 6(11): p. 2567-72.

19. Chawla, L.S., et al., Acute kidney injury and chronic kidney disease as interconnected syndromes. N Engl J Med, 2014. 371(1): p. 58-66.

20. Price, P.M., J. Megyesi, and R.L. Saf Irstein, Cell cycle regulation: repair and regeneration in acute renal failure. Kidney Int, 2004. 66(2): p. 509-14.

21. Yang, L., et al., Epithelial cell cycle arrest in G2/M mediates kidney fibrosis after injury. Nat Med, 2010. 16(5): p. 535-43, 1p following 143.

22. Jun, J.I. and L.F. Lau, Resolution of organ fibrosis. J Clin Invest, 2018. 128(1): p. 97-107.

23. Falke, L.L., et al., Diverse origins of the myofibroblast-implications for kidney fibrosis. Nature Reviews Nephrology, 2015. 11: p. 233.

24. Han, H.I., et al., The role of macrophages during acute kidney injury: destruction and repair. Pediatr Nephrol, 2019. 34(4): p. 561-569.

25. Tang, P.M., D.J. Nikolic-Paterson, and H.Y. Lan, Macrophages: versatile players in renal inflammation and fibrosis. Nat Rev Nephrol, 2019. 15(3): p. 144-158.

26. Kim, M.G., et al., The Role of M2 Macrophages in the Progression of Chronic Kidney Disease following Acute Kidney Injury. PLoS One, 2015. 10(12): p. e0143961.

27. Lee, S., et al., Distinct macrophage phenotypes contribute to kidney injury and repair. J Am Soc Nephrol, 2011. 22(2): p. 317-26.

28. Horowitz, J.C. and V.J. Thannickal, Mechanisms for the Resolution of Organ Fibrosis. Physiology (Bethesda), 2019. 34(1): p. 43-55.

29. Nishida, M., et al., MMP-2 inhibition reduces renal macrophage infiltration with increased fibrosis in UUO. Biochem Biophys Res Commun, 2007. 354(1): p. 133-9.

30. Wang, X., et al., Mice lacking the matrix metalloproteinase-9 gene reduce renal interstitial fibrosis in obstructive nephropathy. Am J Physiol Renal Physiol, 2010. 299(5): p. F973-82.

31. Yang, J., et al., Disruption of tissue-type plasminogen activator gene in mice reduces renal interstitial fibrosis in obstructive nephropathy. J Clin Invest, 2002. 110(10): p. 1525-38.

32. Liu, Y., New insights into epithelial-mesenchymal transition in kidney fibrosis. J Am Soc Nephrol, 2010. 21(2): p. 212-22.

33. Kawamoto, $\mathrm{H}$., et al., Tissue inhibitor of metalloproteinase-3 plays important roles in the kidney following unilateral ureteral obstruction. Hypertens Res, 2006. 29(4): p. 285-94.

34. Zeisberg, M., et al., BMP-7 counteracts TGF- $\beta 1$-induced epithelial-tomesenchymal transition and reverses chronic renal injury. Nature Medicine, 2003. 9(7): p. 964-968. 
35. Turner, J.M., et al., Treatment of chronic kidney disease. Kidney Int, 2012. 81(4): p. 351-62.

36. Naughton, C.A., Drug-induced nephrotoxicity. Am Fam Physician, 2008. 78(6): p. 743-50.

37. Oh, G.S., et al., Cisplatin-induced Kidney Dysfunction and Perspectives on Improving Treatment Strategies. Electrolyte Blood Press, 2014. 12(2): p. 55-65.

38. Kelland, L., The resurgence of platinum-based cancer chemotherapy. Nat Rev Cancer, 2007. 7(8): p. 573-84.

39. Miller, R.P., et al., Mechanisms of Cisplatin nephrotoxicity. Toxins (Basel), 2010. 2(11): p. 2490-518.

40. Oun, R., Y.E. Moussa, and N.J. Wheate, The side effects of platinumbased chemotherapy drugs: a review for chemists. Dalton Trans, 2018. 47(19): p. 6645-6653.

41. Pabla, N. and Z. Dong, Cisplatin nephrotoxicity: mechanisms and renoprotective strategies. Kidney Int, 2008. 73(9): p. 994-1007.

42. Filipski, K.K., et al., Contribution of organic cation transporter 2 (OCT2) to cisplatin-induced nephrotoxicity. Clin Pharmacol Ther, 2009. 86(4): p. 396402.

43. Ciarimboli, G., et al., Organic cation transporter 2 mediates cisplatininduced oto- and nephrotoxicity and is a target for protective interventions. Am J Pathol, 2010. 176(3): p. 1169-80.

44. Sprowl, J.A., et al., Conjunctive therapy of cisplatin with the OCT2 inhibitor cimetidine: influence on antitumor efficacy and systemic clearance. Clin Pharmacol Ther, 2013. 94(5): p. 585-92.

45. Nakamura, T., et al., Disruption of multidrug and toxin extrusion MATE1 potentiates cisplatin-induced nephrotoxicity. Biochem Pharmacol, 2010. 80(11): p. $1762-7$.

46. Townsend, D.M., et al., Metabolism of Cisplatin to a nephrotoxin in proximal tubule cells. J Am Soc Nephrol, 2003. 14(1): p. 1-10.

47. Ozkok, A. and C.L. Edelstein, Pathophysiology of cisplatin-induced acute kidney injury. Biomed Res Int, 2014. 2014: p. 967826.

48. Wei, Q., et al., The pathological role of Bax in cisplatin nephrotoxicity. Kidney Int, 2007. 72(1): p. 53-62.

49. Wei, Q., et al., Activation and involvement of p53 in cisplatin-induced nephrotoxicity. Am J Physiol Renal Physiol, 2007. 293(4): p. F1282-91.

50. Mandic, A., et al., Cisplatin induces endoplasmic reticulum stress and nucleus-independent apoptotic signaling. J Biol Chem, 2003. 278(11): p. 9100-6.

51. Rashid, H.O., et al., ER stress: Autophagy induction, inhibition and selection. Autophagy, 2015. 11(11): p. 1956-1977.

52. Lin, T.A., V.C. Wu, and C.Y. Wang, Autophagy in Chronic Kidney Diseases. Cells, 2019. 8(1).

53. Wang, Z. and M.E. Choi, Autophagy in kidney health and disease. Antioxid Redox Signal, 2014. 20(3): p. 519-37. 
54. Ramesh, G. and W.B. Reeves, TNF-alpha mediates chemokine and cytokine expression and renal injury in cisplatin nephrotoxicity. J Clin Invest, 2002. 110(6): p. 835-42.

55. Sharp, C.N. and L.J. Siskind, Developing better mouse models to study cisplatin-induced kidney injury. Am J Physiol Renal Physiol, 2017. 313(4): p. F835-F841.

56. Skinner, R., et al., Persistent nephrotoxicity during 10-year follow-up after cisplatin or carboplatin treatment in childhood: relevance of age and dose as risk factors. Eur J Cancer, 2009. 45(18): p. 3213-9.

57. Sharp, C.N., et al., Subclinical kidney injury induced by repeated cisplatin administration results in progressive chronic kidney disease. Am J Physiol Renal Physiol, 2018. 315(1): p. F161-F172.

58. Sharp, C.N., et al., Repeated administration of low-dose cisplatin in mice induces fibrosis. Am J Physiol Renal Physiol, 2016. 310(6): p. F560-8.

59. Shayman, J.A., Sphingolipids. Kidney Int, 2000. 58(1): p. 11-26.

60. Abou Daher, A., et al., Translational Aspects of Sphingolipid Metabolism in Renal Disorders. Int J Mol Sci, 2017. 18(12).

61. Dupre, T.V. and L.J. Siskind, The role of sphingolipids is acute kidney injury. Adv Biol Regul, 2018. 70: p. 31-39.

62. Dupre, T.V., et al., Inhibiting glucosylceramide synthase exacerbates cisplatin-induced acute kidney injury. J Lipid Res, 2017. 58(7): p. 14391452.

63. Bajwa, A., et al., Sphingosine 1-phosphate receptor-1 enhances mitochondrial function and reduces cisplatin-induced tubule injury. J Am Soc Nephrol, 2015. 26(4): p. 908-25.

64. Perry, H.M., et al., Endothelial Sphingosine 1Phosphate Receptor1 Mediates Protection and Recovery from Acute Kidney Injury. J Am Soc Nephrol, 2016. 27(11): p. 3383-3393.

65. Bartels, K., A. Grenz, and H.K. Eltzschig, Sphingosine-1-phosphate receptor signaling during acute kidney injury: the tissue is the issue. Kidney Int, 2014. 85(4): p. 733-5.

66. Coant, N., et al., Ceramidases, roles in sphingolipid metabolism and in health and disease. Adv Biol Regul, 2017. 63: p. 122-131.

67. Novgorodov, S.A., et al., Essential roles of neutral ceramidase and sphingosine in mitochondrial dysfunction due to traumatic brain injury. $\mathrm{J}$ Biol Chem, 2014. 289(19): p. 13142-54.

68. Geoffroy, K., et al., Glomerular proliferation during early stages of diabetic nephropathy is associated with local increase of sphingosine-1-phosphate levels. FEBS Lett, 2005. 579(5): p. 1249-54.

69. Sundaram, K., et al., Loss of neutral ceramidase protects cells from nutrient- and energy -deprivation-induced cell death. Biochem J, 2016. 473(6): p. 743-55.

70. Takahashi, A., et al., Autophagy guards against cisplatin-induced acute kidney injury. Am J Pathol, 2012. 180(2): p. 517-25. 
71. Tani, M., et al., Specific and sensitive assay for alkaline and neutral ceramidases involving C12-NBD-ceramide. J Biochem, 1999. 125(4): p. 746-9.

72. Mancinelli, R., et al., Multifaceted Roles of GSK-3 in Cancer and Autophagy-Related Diseases. Oxid Med Cell Longev, 2017. 2017: p. 4629495.

73. Marchand, B., et al., Glycogen synthase kinase-3 (GSK3) inhibition induces prosurvival autophagic signals in human pancreatic cancer cells. J Biol Chem, 2015. 290(9): p. 5592-605.

74. Grgic, I., et al., Targeted proximal tubule injury triggers interstitial fibrosis and glomerulosclerosis. Kidney Int, 2012. 82(2): p. 172-83.

75. Nam, S.A., et al., Autophagy attenuates tubulointerstital fibrosis through regulating transforming growth factor $\beta$ and NLRP3 inflammasome signaling pathway. Cell Death \& Disease, 2019. 10(2): p. 78.

76. Livingston, M.J., et al., Persistent activation of autophagy in kidney tubular cells promotes renal interstitial fibrosis during unilateral ureteral obstruction. Autophagy, 2016. 12(6): p. 976-98.

77. Corazzari, M., et al., Endoplasmic Reticulum Stress, Unfolded Protein Response, and Cancer Cell Fate. Front Oncol, 2017. 7: p. 78.

78. Rutkowski, D.T. and R.J. Kaufman, That which does not kill me makes me stronger: adapting to chronic ER stress. Trends Biochem Sci, 2007. 32(10): p. 469-76.

79. Maekawa, H. and R. Inagi, Stress Signal Network between Hypoxia and ER Stress in Chronic Kidney Disease. Front Physiol, 2017. 8: p. 74.

80. Shu, S., et al., Endoplasmic reticulum stress is activated in post-ischemic kidneys to promote chronic kidney disease. EBioMedicine, 2018. 37: p. 269-280. 


\title{
CURRICULUM VITAE
}

\author{
Sophia M. Sears \\ University of Louisville School of Medicine \\ Department of Pharmacology \& Toxicology \\ 505 South Hancock Street (CTRB 252 G) \\ Louisville, KY 40202 \\ Email: smsear03@louisville.edu
}

Phone: (812)653-9691

\section{Education:}

B.A. Molecular Biology \& Biochemistry (GPA 3.9)

2013-2017

Goshen College, Department of Biological Sciences

Goshen, Indiana

\section{Honors and Awards:}

General Chemistry Award (Best GPA in class), Goshen College 2013-2014

Dean's List, Goshen College

2013-2017

Alicia Showalter Reynolds, Women in Science Scholarship

2015-2016

NAIA Daktronics Scholar-Athlete Award

NAIA DII Women's Basketball All American

Graduated Summa Cum Lade, Goshen College

IPIBS Fellowship (full tuition and stipend), University of Louisville

2017-2019

\section{Research Experience:}

August 2017- Present

Graduate Student, University of Louisville, Department of Pharmacology \& Toxicology Louisville, KY- Characterizing the role of neutral ceramidase in 
cisplatin-induced kidney injury. Lab mentors: Dr. Leah Siskind and Dr. Levi Beverly

June 2016- July 2016

Research Intern, R25 Cancer Education Program, University of Louisville, Louisville, KY- Evaluating effects of therapeutic compounds on cadmium-induced prostate cancer. Lab mentors: Dr. Chendil Damodaran and Dr. Jonathan Freedman

August 2015- May 2016

Independent Research/Research Assistant, Goshen College, Department of Biological Sciences, Goshen, IN- Determining a role for unc-53/Nav2 in cholinergic signaling in Caenorhabditis elegans. Lab mentors: Dr. Kristopher Schmidt

\section{August 2013- December 2013}

Research Assistant, Goshen College, Department of Biological Sciences, Goshen, IN- Data analysis and lab assistant in evaluating synaptic efficacy of the amphibian neuromuscular junction. Lab mentor: Dr. Rich Manalis

\section{Teaching Experience:}

August 2014- December 2014

Teaching Assistant, Goshen College, Department of Chemistry, Goshen, INAssisted in grading, instruction, laboratory preparation, and lab supervision for Chemistry 101, Chemistry of Life. Course instructor: Dr. Daniel Smith

\section{Oral Presentations:}

Neutral ceramidase inhibition as a potential treatment for acute kidney injury. March 2018. Pharmacology \& Toxicology William J. Waddell Seminar Series. University of Louisville, Louisville, KY.

Evaluating functions and interactions of unc53/Nav2 in cholinergic signaling in Caenorhabditis elegans. April 2017. BIOL 410: Biology Senior Seminar. Goshen College, Goshen, IN.

Evaluating effects of therapeutic compounds on cadmium-induced prostate cancer. January 2017. Science Speakers Lecture Series. Goshen College, Goshen, IN.

Determining a role for unc53/Nav2 in cholinergic signaling in Caenorhabditis elegans. December 2015. BIOL 331: Biology Junior Seminar. Goshen College, Goshen, IN.

Synthesis and discovery of triazolopyridazine PC25. April 2015. CHEM 302 :

Organinc Chemistry. Goshen College, Goshen, IN. 


\section{Research Posters:}

Sears SM, Sharp C, Saforo D, Doll M, and Siskind L. Neutral ceramidase inhibition as a potential treatment for cisplatin-induced kidney injury. October 2018. Researh!Louisville. University of Louisville, Louisville, KY. Link in progress.

Sears SM, Pal D, Kolluru V, Damodaran C, and Freedman J. Effects of therapeutic compounds on cadmium-induced prostate cancer. October 2016. Research!Louisville. University of Louisville, Louisville, KY. Link: http://louisville.edu/medicine/departments/pharmacology/research/nci-r25cancer-education/2016-ug-posters-22-28

\section{Peer Reviewed Publications:}

Pal D, Suman S, Kolluru V, Sears S, Das TP, Alatassi PH, Ankem MK, Freedman JH, and Damodaran C. (2017). "Inhibition of autophagy prevents cadmium-induced prostate carcinogenesis." British Journal of Cancer, 177: 56-64 doi: 10.1038/bjc.2017.143

\section{Conferences/Workshops Attended:}

Society of Toxicologic Pathology Annual Symposium: Keeping it Renal. June 2018. Indianapolis, IN.

UAB-UCSD O'Brien Center Workshop on Flow Cytometry for the Nephrology Researcher. October 2018. Birmingham, AL.

\section{Service and Outreach:}

Central America Study and Service Term, Matagalpa, Nicaragua. Escuela Especial La Amistad: Public school for children with disabilities. May-August 2015. Volunteered in classroom for disabled children ages 5-8. Developed basic skills understanding and speaking Spanish.

Paoli Community Schools, Paoli, IN. Moderate to Severe Special Education Grades K-6. August-December 2008. Assisted teachers in classroom and helped students complete daily tasks.

Paoli Mennonite Fellowship, Paoli, IN. Performed regular service through participation in the church youth group:

- Re-shingled roofs of hurricane victims in Mashulaville, MS, March 2008

- Provided lawn care for senior citizens, 2006-2008

- Cleanup of local county roads, 2005 